\title{
THE RECOMBINATION EQUATION FOR INTERVAL PARTITIONS
}

\author{
MICHAEL BAAKE AND ELHAM SHAMSARA
}

\begin{abstract}
The general deterministic recombination equation in continuous time is analysed for various lattices, with special emphasis on the lattice of interval (or ordered) partitions. Based on the recently constructed [5] general solution for the lattice of all partitions, the corresponding solution for interval partitions is derived and analysed in detail. We focus our attention on the recursive structure of the solution and its decay rates, and also discuss the solution in the degenerate cases, where it comprises products of monomials with exponentially decaying factors. This can be understood via the Markov generator of the underlying partitioning process that was recently identified. We use interval partitions to gain insight into the structure of the solution, while our general framework works for arbitrary lattices.
\end{abstract}

\section{INTRODUCTION}

Recombination is an important mechanism in population genetics. It is one of the fundamental processes that underlies the time evolution of a population of individuals under sexual reproduction; see [9, 13, 11] for general background and [12, 8, 10] for important contributions. Recombination is a stochastic process that, in continuous time and in the deterministic limit of large populations, leads to a nonlinear ordinary differential equation (ODE) in the Banach space of finite measures on a locally compact product space for the types that characterise the individuals; see [7, 5] and references therein. The flow of this ODE generally acts on an infinite-dimensional space, but has a number of nice properties, including preservation of positivity under the forward flow as well as norm preservation in the positive sector.

Moreover, the recombination ODE admits a reduction to a finite-dimensional nonlinear system of ODEs via a suitable ansatz in form of a finite convex combination of probability measures that derive from the initial condition by the application of a finite set of (also nonlinear) operators, which are known as recombinators. The explicit solution of this ODE system was recently achieved in [5], building on previous work [7, 6, 3, 15, in a recursive way. The solution formula derived there was explicit enough to establish the exponentially fast convergence of the solution to a unique equilibrium that only depends on the initial condition, as expected from the previously known cases [7] as well as the general theory [9]; see also the indroduction of [5] and references therein for more.

The mathematical setting was based on the lattice $\mathbb{P}(S)$ of all partitions of a finite set, say $S=\{1,2, \ldots, n\}$, and was not restricted to the biologically motivated case of two parents, but

2010 Mathematics Subject Classification. 34G20, 06B23, 92D10, 60J25.

Key words and phrases. Recombination equation, population genetics, Markov generator, interval partitions, measure-valued equations, nonlinear ODEs, closed solution. 
also allowed for an arbitrary recombination scheme. This results in an interesting nonlinear ODE system in its own right, and adds a nontrivial example of a solvable case. Nevertheless, the treatment in [5] did not consider other lattices and thus did not explore the full structure of the solution in lattice-theoretic terms. It is the aim of the present paper to continue this development, in particular by employing the lattice of ordered or interval partitions. This has the advantage that complete closed expressions can be derived for sizes up to four sites, and with some effort even for five sites. This way, the intricate and rather complex nature of the recursive approach becomes more transparent, and a better understanding of the solution is possible. Also, we are able to shed more light on the non-generic degenerate cases with singularities in the recursion, which were only briefly looked at in [5] and had been excluded from consideration in all previous attempts [13, 10]. Finally, motivated by the underlying partitioning process from [5, Sec. 6] and its Kolmogorov backward and forward equations, we derive a linear ODE system for the solution of the recombination ODE, thus explaining why the nonlinear ODE was solvable in the first place and how the degeneracies emerge.

The paper is organised as follows. In Section 2, we introduce the mathematical concepts from lattice theory that we need, with some focus on our later applications (with one result of general interest being added as an Appendix). This is followed by a review of some of the known results for the recombination ODE in Section 3, adapted to our more general setting. This section also includes some new results on the decay rates of the system. Then, we consider the ODE for the lattice $\mathbb{I}(S)$ of interval partitions in some detail in Section 4. This lattice allows to explore the general solution without the combinatorial complexity of the full lattice $\mathbb{P}(S)$, and leads to a number of previously unknown properties. Special cases (with up to five sites) are written out in explicit form (and with possible applications in mind) in Sections 5 and 6, before we sketch further extensions and directions in Section 7, including the derivation of a linear ODE system for the recombination process.

\section{Preliminaries}

Let $S$ be a finite set, for instance $S=\{1,2, \ldots, n\}$, and let $\mathbb{P}(S)$ denote the lattice of all partitions of $S$, with order relation $\preccurlyeq$, so that $\mathcal{A} \preccurlyeq \mathcal{B}$ stands for $\mathcal{A}$ being a refinement of $\mathcal{B}$. Below, we use the shorthands $\underline{1}=\{S\}$ and $\underline{0}=\{\{1\},\{2\}, \ldots,\{n\}\}$ for the maximal and the minimal element of $\mathbb{P}(S)$. We use $\mathcal{A} \wedge \mathcal{B}$ for the coarsest refinement and $\mathcal{A} \vee \mathcal{B}$ for the finest coarsening of $\mathcal{A}$ and $\mathcal{B}$.

Let $\mathbb{V} \subseteq \mathbb{P}(S)$ denote a sublattice. It is called decomposable when there is a partition $S=S_{1} \cup S_{2}$ of $S$ into two non-empty subsets such that $\mathbb{V} \subseteq \mathbb{P}\left(S_{1}\right) \times \mathbb{P}\left(S_{2}\right)$. Moreover, $\mathbb{V}$ is called reducible when a subset $U \subset S$ exists such that $\mathbb{V}$ is isomorphic with a sublattice of $\mathbb{P}(U)$. In this case, one may find a partition $\left\{A_{1}, A_{2}, \ldots, A_{r}\right\} \in \mathbb{P}(S)$, with $r<n$, such that $\mathbb{V}$ can be identified with a sublattice of $\mathbb{P}(\{1,2, \ldots, r\})$.

Clearly, we are primarily interested in lattices $\mathbb{V}$ that are both indecomposable and irreducible, which implies that $\underline{0}, \underline{1} \in \mathbb{V}$. The perhaps most important cases are $\mathbb{P}(S)$ itself and $\mathbb{I}(S)$, the sublattice of ordered or interval partitions. The latter are the partitions 
$\mathcal{A}=\left\{A_{1}, A_{2}, \ldots, A_{r}\right\}$ such that all parts $A_{i}$ are contiguous subsets of $S$, so

$$
\mathcal{A}=\left\{\left\{1, \ldots, k_{1}\right\},\left\{k_{1}+1, \ldots, k_{2}\right\}, \ldots,\left\{k_{r-1}+1, \ldots, n\right\}\right\} .
$$

Another interesting lattice consists of all non-crossing partitions.

Apart from our lattice $\mathbb{V}$ for the set $S$, we will need the induced lattices for all $\varnothing \neq U \subseteq S$. They are defined by

$$
\mathbb{V}(U):=\left\{\left.\mathcal{A}\right|_{U} \mid \mathcal{A} \in \mathbb{V}\right\}
$$

where $\left.\mathcal{A}\right|_{U}$ denotes the restriction of $\mathcal{A}$ to $U$. It consists of all non-empty sets of the form $A_{i} \cap U$ as its parts. With this notation, one has $\mathbb{V}=\mathbb{V}(S)$. We write $|\mathcal{A}|$ for the number of parts of a partition $\mathcal{A}$, and $\operatorname{supp}(\mathcal{A}):=\bigcup_{i=1}^{|\mathcal{A}|} A_{i}$ for the underlying set that is partitioned.

In our setting, we also have a non-negative function

$$
\varrho: \mathbb{P}(S) \longrightarrow \mathbb{R}_{\geqslant 0},
$$

whose values $\varrho(\mathcal{A})$ for $\mathcal{A} \in \mathbb{P}(S)$ have the meaning of recombination rates in the underlying continuous dynamical system we want to study. Of particular relevance is the sublattice

$$
\mathbb{V}:=\langle\mathcal{A} \in \mathbb{P}(S) \mid \varrho(\mathcal{A})>0\rangle
$$

which is the smallest sublattice of $\mathbb{P}(S)$ that contains all partitions with strictly positive rates. To guarantee that $\mathbb{V}$ is both irreducible and indecomposable, we will usually assume that it contains all relevant partitions with two parts.

It will be vital to our later analysis that quantities defined on $\mathbb{V}(S)$ have consistent counterparts on all induced sublattices for non-empty subsets $U \subset S$. For the recombination rates, this is done via

$$
\varrho^{U}(\mathcal{A}):=\sum_{\substack{\left.\mathcal{B} \in \mathbb{V}(S) \\ \mathcal{B}\right|_{U}=\mathcal{A}}} \varrho^{S}(\mathcal{B}) .
$$

This 'top down' formula is consistent with the standard marginalisation of probability vectors $q^{S}$ for the top system to probability vectors $q^{U}$ for the subsystem defined by $U$; see [5, Sec. 5] for a detailed account.

One quantity that requires a different approach is the decay rate of a partition, which needs a 'bottom up' recursion. The decay rates $\psi=\psi^{S}$ are defined recursively via those of the subsystems for non-empty $U \subseteq S$. One has [3, 5]

$$
\psi^{U}(\underline{1})=\sum_{\mathcal{A} \neq \underline{1}} \varrho^{U}(\mathcal{A})
$$

which implies $\psi^{U}(\underline{1})=0$ whenever $|U|=1$, together with the recursion

$$
\psi^{U}(\mathcal{A}):=\sum_{i=1}^{|\mathcal{A}|} \psi^{A_{i}}\left(\left.\underline{1}\right|_{A_{i}}\right)
$$

for any partition $\mathcal{A}=\left\{A_{1}, A_{2}, \ldots, A_{|\mathcal{A}|}\right\} \in \mathbb{V}(U)$. In particular, one always has $\psi^{U}(\underline{0})=0$. 
Functions such as $\varrho$ or $\psi$ can be viewed as elements of the Möbius algebra $\mathbb{M}_{\mathbb{R}}(\mathbb{V})$ for $\mathbb{V}$ over the field $\mathbb{R}$. This is the free real vector space with basis $\left\{\varepsilon_{\mathcal{A}} \mid \mathcal{A} \in \mathbb{V}\right\}$, where $\varepsilon_{\mathcal{A}}$ is the function given by $\varepsilon_{\mathcal{A}}(\mathcal{B}):=\delta_{\mathcal{A}, \mathcal{B}}$. Then, multiplication is defined by $\varepsilon_{\mathcal{A}} \cdot \varepsilon_{\mathcal{B}}=\varepsilon_{\mathcal{A}} \delta_{\mathcal{A}, \mathcal{B}} ;$ compare [2, Sec. IV.4.A] for details. More important to us is the real incidence algebra [2, Ch. IV.1]

$$
\mathbb{A}_{\mathbb{R}}(\mathbb{V}):=\{f: \mathbb{V} \times \mathbb{V} \longrightarrow \mathbb{R} \mid f(\mathcal{A}, \mathcal{B})=0 \text { whenever } \mathcal{A} \npreceq \mathcal{B}\} .
$$

Here, addition and multiplication with a real number are defined as usual, while multiplication $f * g$ is defined via the (generally non-commutative) convolution

$$
(f * g)(\mathcal{A}, \mathcal{B})=\sum_{\mathcal{A} \preccurlyeq \mathcal{C} \preccurlyeq \mathcal{B}} f(\mathcal{A}, \mathcal{C}) g(\mathcal{C}, \mathcal{B}),
$$

where we follow 22 to mark the summation variable by placing a dot underneath it. Note that this gives $(f * g)(\mathcal{A}, \mathcal{B})=0$ for $\mathcal{A} \npreceq \mathcal{B}$. Clearly, $\delta(\mathcal{A}, \mathcal{B})=\delta_{\mathcal{A}, \mathcal{B}}$ defines an element $\delta \in \mathbb{A}_{\mathbb{R}}(\mathbb{V})$ that is the neutral element for multiplication, so $\delta * f=f * \delta=f$ for all $f \in \mathbb{A}_{\mathbb{R}}(\mathbb{V})$.

Recall that an element $f \in \mathbb{A}_{\mathbb{R}}(\mathbb{V})$ is a unit (or invertible) when some $g \in \mathbb{A}_{\mathbb{R}}(\mathbb{V})$ exists such that $f * g=g * f=\delta$. This happens if and only if $f(\mathcal{A}, \mathcal{A}) \neq 0$ for all $\mathcal{A} \in \mathbb{V}$, and the inverse is then unique, hence written as $f^{-1}$. The left inverse property gives $f^{-1}(\mathcal{A}, \mathcal{A})=1 / f(\mathcal{A}, \mathcal{A})$ together with the recursion

$$
f^{-1}(\mathcal{A}, \mathcal{B})=\frac{-1}{f(\mathcal{B}, \mathcal{B})} \sum_{\mathcal{A} \preccurlyeq \mathcal{C} \prec \mathcal{B}} f^{-1}(\mathcal{A}, \mathcal{C}) f(\mathcal{C}, \mathcal{B})
$$

for $\mathcal{A} \prec \mathcal{B}$. A similar recursion follows from the right inverse property. In the Appendix, we add a classic (but less well-known) non-recursive formula [16], which is sometimes of advantage. An important unit is the zeta function $\zeta$, defined by $\zeta(\mathcal{A}, \mathcal{B})=1$ for $\mathcal{A} \preccurlyeq \mathcal{B}$ and by $\zeta(\mathcal{A}, \mathcal{B})=0$ otherwise. Its inverse, $\mu=\zeta^{-1}$, is the Möbius function of the lattice, which plays a central role in many (if not most) lattice-theoretic calculations; compare [2, Prop. 4.6]. It is given by $\mu(\mathcal{A}, \mathcal{A})=1$ for all $\mathcal{A} \in \mathbb{V}$ together with

$$
\mu(\mathcal{A}, \mathcal{B})=-\sum_{\mathcal{A} \preccurlyeq \mathcal{C} \prec \mathcal{B}} \mu(\mathcal{A}, \mathcal{C})=-\sum_{\mathcal{A} \prec \mathcal{C} \preccurlyeq \mathcal{B}} \mu(\mathcal{C}, \mathcal{B})
$$

for $\mathcal{A} \prec \mathcal{B}$. It can often also be given in closed form, which will be used below.

\section{Recombinaton EQUATions}

The ingredients for the formulation of the recombination ODE are a finite set $S$ together with an irreducible and indecomposable lattice $\mathbb{V}$ of partitions of $S$, a general product space $X=\times_{i \in S} X_{i}$ with all $X_{i}$ locally compact, the Banach space $\mathcal{M}(X)$ of finite Borel measures on $X$ with total variation norm $\|$.$\| , and the set of nonlinear recombinators R_{\mathcal{A}}$ for $\mathcal{A} \in \mathbb{V}$, defined by

$$
R_{\mathcal{A}}(\omega)=\frac{1}{\|\omega\|^{|\mathcal{A}|-1}} \bigotimes_{i=1}^{|\mathcal{A}|}\left(\pi_{A_{i}} \cdot \omega\right)
$$


for $\omega \in \mathcal{M}(X)$. On the positive cone $\mathcal{M}_{+}(X)$, the recombinators satisfy $R_{\mathcal{A}} R_{\mathcal{B}}=R_{\mathcal{A} \wedge \mathcal{B}}$ for $\mathcal{A}, \mathcal{B} \in \mathbb{V}$. Note that one can define a matching order relation among them by saying that $R_{\mathcal{A}} \preccurlyeq R_{\mathcal{B}}$ precisely when $R_{\mathcal{A}} R_{\mathcal{B}}=R_{\mathcal{A}}$. Consequently, one has $R_{\mathcal{A}} \preccurlyeq R_{\mathcal{B}}$ if and only if $\mathcal{A} \preccurlyeq \mathcal{B}$.

The recombination ODE now reads [7, 6, [5]

$$
\dot{\omega}_{t}=\sum_{\mathcal{A} \in \mathbb{V}} \varrho(\mathcal{A})\left(R_{\mathcal{A}}-\mathbb{1}\right)\left(\omega_{t}\right)
$$

with non-negative numbers $\varrho(\mathcal{A})=\varrho^{S}(\mathcal{A})$ as introduced above. The forward flow preserves the positive cone of $\mathcal{M}(X)$ and, within it, also the norm of the initial condition, $\omega_{0}$ say, see [5] and references therein for details. Since this is also the setting for the dynamics of recombination, we restrict our attention to probability measures on $X$ (denoted by $\mathcal{P}(X)$ ) as initial conditions from now on.

The key to solve Eq. (4), with $\omega_{0} \in \mathcal{P}(X)$, is the decomposition

$$
\omega_{t}=\sum_{\mathcal{A} \in \mathbb{V}} a_{t}(\mathcal{A}) R_{\mathcal{A}}\left(\omega_{0}\right)
$$

which effectively separates the dependence on the initial condition from the time evolution. Here, the coefficients $a_{t}$ constitute a family of probability vectors on $\mathbb{V}$ with initial condition $a_{0}(\mathcal{A})=\delta(\mathcal{A}, \underline{1})$. As a result of [5, Lemma 2 and Prop. 7], the induced ODE for the coefficients then reads

$$
\dot{a}_{t}(\mathcal{A})=-\psi(\underline{1}) a_{t}(\mathcal{A})+\sum_{\mathcal{A} \preccurlyeq \mathcal{B} \prec \underline{1}} \varrho(\mathcal{B}) \prod_{i=1}^{|\mathcal{B}|} a_{t}^{B_{i}}\left(\left.\mathcal{A}\right|_{B_{i}}\right),
$$

where $a_{t}^{U}$ is the marginalised probability vector on the sublattice $\mathbb{V}(U)$. Note that this vector has a clear meaning in terms of a (stochastic) partitioning process, as explained in detail in [5, Sec. 6]. Each $a_{t}^{U}$ satisfies an ODE of the same type as in Eq. (66) for the subsystem defined by $\varnothing \neq U \subseteq S$; compare [5, Sec. 5] for details on the important marginalisation consistency of our equations. A step-by-step repetition of the results from [7, 6, 5] in our more general lattice-theoretic setting now leads to the following fundamental result.

Theorem 1. The Cauchy problem defined by the $O D E$ (4) with initial condition $\omega_{0} \in \mathcal{M}(X)$ has a unique solution. The corresponding flow in forward time preserves the space $\mathcal{P}(X)$ of probability measures on $X$. For any $\omega_{0} \in \mathcal{P}(X)$, the solution is of the form (15), where the coefficients $a_{t}$ constitute probability vectors on $\mathbb{V}$ that are the unique solution of the nonlinear ODE system (6) with initial condition $a_{0}(\mathcal{A})=\delta(\mathcal{A}, \underline{1})$.

The main progress of [5] in comparison to previous work is the insight that, for generic values of the recombination rates $\varrho$, an explicit recursive formula for the solution can be derived. In our present setting, it can be formulated as follows. Let $\psi^{U}$ be the rate functions defined above, and set

$$
a_{t}^{U}(\mathcal{A}):=\sum_{\mathcal{B} \succcurlyeq \mathcal{A}} \theta^{U}(\mathcal{A}, \mathcal{B}) \mathrm{e}^{-\psi^{U}(\mathcal{B}) t}
$$


for $\mathcal{A} \in \mathbb{V}(U)$ and all non-empty $U \subseteq S$, where $\theta^{U}$ is an element of the incidence algebra $\mathbb{A}_{\mathbb{R}}(\mathbb{V}(U))$. Now, let $\theta^{U}(\underline{1}, \underline{1})=1$ together with

$$
\theta^{U}(\mathcal{A}, \underline{1})=-\sum_{\mathcal{A} \preccurlyeq \mathcal{B} \prec \underline{1}} \theta^{U}(\mathcal{A}, \mathcal{B})
$$

for any $\mathcal{A} \prec \underline{1}$, which is just a reflection of the initial conditions we need. Inserting Eq. (7) into the ODE (6) and observing the marginalisation consistency then leads to the recursion

$$
\theta^{U}(\mathcal{A}, \mathcal{B})=\sum_{\mathcal{B} \preccurlyeq \mathcal{C} \prec \underline{1}} \frac{\varrho^{U}(\mathcal{C})}{\psi^{U}(\underline{1})-\psi^{U}(\mathcal{B})} \prod_{i=1}^{|\mathcal{C}|} \theta^{C_{i}}\left(\left.\mathcal{A}\right|_{C_{i}},\left.\mathcal{B}\right|_{C_{i}}\right)
$$

for all $\mathcal{A} \preccurlyeq \mathcal{B} \prec \underline{1}$, provided that no denominator vanishes and that the exponential functions in Eq. (7) are linearly independent. The entire analysis from [5] for the lattice $\mathbb{P}(S)$ remains valid for our irreducible and indecomposable lattice $\mathbb{V}$, and leads to the following result.

Theorem 2. Let $S$ be a finite set, and $\mathbb{V}=\mathbb{V}(S)$ an irreducible, indecomposable lattice of partitions of $S$. Assume that the decay rates $\psi^{U}(\mathcal{A})$ are recursively defined for $\varnothing \neq U \subseteq S$ according to Eqs. (2) and (3), with the induced recombination rates $\varrho^{U}$ on subsystems being given by Eq. (11). Assume further that the decay rates $\psi^{S}(\mathcal{A})$ with $\mathcal{A} \in \mathbb{V}$ are distinct.

Then, the exponential ansatz (7) solves the Cauchy problem of Eq. (6) with initial condition $a_{t}^{U}(\mathcal{A})=\delta(\mathcal{A}, \underline{1})$ for all $\varnothing \neq U \subseteq S$ if and only if the coefficients $\theta^{U}$ are recursively determined by Eq. (9) together with $\theta^{U}(\underline{1}, \underline{1})=1$ and Eq. (8)).

Sketch of proof. Once again, this is a step-by-step transfer of the arguments for the lattice $\mathbb{P}(S)$ from [5] to $\mathbb{V} \subset \mathbb{P}(S)$. Irreducibility and indecomposability of $\mathbb{V}$ ensure that the latter contains $\underline{0}$ and $\underline{1}$, which also implies the corresponding statement for $\mathbb{V}(U)$ and all $U$ under consideration.

In particular, given $\varnothing \neq U \subseteq S$, the distinctness of all $\psi^{U}(\mathcal{B})$ with $\mathcal{B} \in \mathbb{V}(U)$ follows from the assumed distinctness of the $\psi^{S}(\mathcal{A})$ with $\mathcal{A} \in \mathbb{V}$ by an application of [5, Lemma 5], which holds in the generality we need here. This allows to use the ansatz (7) together with a comparison of coefficients to conclude as claimed, where all appearing denominators are non-zero as a consequence of the distinctness of the decay rates on all levels.

The condition on the distinctness of the decay rates $\psi^{S}(\mathcal{A})$ with $\mathcal{A} \in \mathbb{V}$ covers the generic case (both topologically and measure-theoretically). When looking at the excluded cases, one realises that some degeneracies are 'harmless' in the sense that the solution formula (7) remains valid (which also means that the selected exponential functions still suffice), while there are also 'bad' degeneracies that lead to singularities in the recursion and render this approach incomplete (due to the necessity of further functions beyond simple exponentials; see [5, Sec. 9 and Appendix]). Below, we shall discuss this phenomenon in detail for $\mathbb{V}=\mathbb{I}(S)$, and later derive an alternative explanation in terms of Jordan matrices for linear ODE systems. At this stage, we recall [5, Cor. 6] and state it for our more general situation as follows. 
Corollary 1. Let $S$ and $\mathbb{V}$ be as in Theorem 2, Then, the generic recursive solution extends to all recombination rates $\varrho^{S}(\mathcal{A})$, with $\mathcal{A} \in \mathbb{V}$, such that $\psi^{U}(\underline{1}) \neq \psi^{U}(\mathcal{B})$ holds for all $\varnothing \neq U \subseteq S$ and all $\mathcal{B} \in \mathbb{V}(U)$.

In [5], we also defined a linear reference system for $\mathbb{V}=\mathbb{P}(S)$. The definition extends to any lattice $\mathbb{V}$ that contains $\underline{1}$ as

$$
\chi(\mathcal{A})=\sum_{\mathcal{B} \notin[\mathcal{A}, \underline{1}]} \varrho(\mathcal{B}) \quad \text { with } \quad[\mathcal{A}, \underline{1}]:=\{\mathcal{C} \in \mathbb{V} \mid \mathcal{A} \preccurlyeq \mathcal{C} \preccurlyeq \underline{1}\}
$$

where it is understood that we sum over the partitions of $\mathbb{V}$, subject to the condition specified, and analogously for the subsystems defined by the non-empty subsets $U \subseteq S$. In particular, one always has $\chi(\underline{1})=\psi(\underline{1})$. Then, a simple calculation leads to the following result.

Fact 1. If $\underline{1} \in \mathbb{V}$, the decay rates $\chi$ of $E q$. (10) satisfy the relation

$$
\chi(\underline{1})-\chi(\mathcal{A})=\sum_{\mathcal{A} \preccurlyeq \mathcal{B} \prec \underline{1}} \varrho(\mathcal{B}) .
$$

Moreover, if $\mathbb{V}=\mathbb{I}(S)$ and if $\varrho(\mathcal{A})>0$ holds for all $\mathcal{A}$ with two parts, one has

$$
\chi(\underline{1})-\chi(\mathcal{A})>0
$$

for all interval partitions $\mathcal{A} \neq \underline{1}$.

Whenever $|U| \leqslant 3$, we had $\psi^{U}=\chi^{U}$, and one has $\psi^{U}(\underline{1})=\chi^{U}(\underline{1})$ for any $U \neq \varnothing$, while no general relation was derived in [5]. To formulate one, we say that a partition $\mathcal{B}$ splits a set $V \subseteq U$ when the restriction $\left.\mathcal{B}\right|_{V}$ has at least two parts. It is then clear what it means to say that $\mathcal{B}$ splits one or more parts of another partition $\mathcal{A}$. Note that $\mathcal{B}$ can split a part of $\mathcal{A}$ without satisfying $\mathcal{B} \preccurlyeq \mathcal{A}$.

This notation allows us to rewrite Eq. (10) for $\mathbb{V}=\mathbb{V}(S)$ as

$$
\chi(\mathcal{A})=\sum_{\mathcal{B} \notin[\mathcal{A}, 1]} \varrho(\mathcal{B})=\sum_{\begin{array}{c}
\mathcal{B} \text { splits at least } \\
\text { one part of } \mathcal{A}
\end{array}} \varrho(\mathcal{B})=\sum_{n \geqslant 1} \sum_{\begin{array}{c}
\mathcal{B} \text { splits } n \\
\text { parts of } \mathcal{A}
\end{array}} \varrho(\mathcal{B}),
$$

where all partition sums run over $\mathbb{V}$. The analogous relation also holds for all $\mathbb{V}(U)$ with $\varnothing \neq U \subset S$. Observe that the last identity implies the relation

$$
\chi^{U}(\underline{1})=\sum_{\underline{1} \neq \mathcal{A} \in \mathbb{V}(U)} \varrho^{U}(\mathcal{A})=\sum_{\underline{1} \neq \mathcal{A} \in \mathbb{V}(U)} \sum_{\substack{\left.\mathcal{B} \in \mathbb{V} \\ \mathcal{B}\right|_{U}=\mathcal{A}}} \varrho(\mathcal{B})=\sum_{\substack{\mathcal{B} \in \mathbb{V} \\ \mathcal{B} \text { splits } U}} \varrho(\mathcal{B}) .
$$


Now, we can rewrite $\psi$ as

$$
\begin{aligned}
\psi(\mathcal{A}) & =\sum_{i=1}^{|\mathcal{A}|} \psi^{A_{i}}\left(\left.\underline{1}\right|_{A_{i}}\right)=\sum_{i=1}^{|\mathcal{A}|} \chi^{A_{i}}\left(\left.\underline{1}\right|_{A_{i}}\right)=\sum_{i=1}^{|\mathcal{A}|} \sum_{\substack{\mathcal{B} \text { splits } \\
\text { at least } A_{i}}} \varrho(\mathcal{B}) \\
& =\sum_{i=1}^{|\mathcal{A}|}\left(\sum_{\substack{\mathcal{B} \text { splits } \\
\text { only } A_{i}}} \varrho(\mathcal{B})+\sum_{\substack{\mathcal{B} \text { splits } A_{i} \text { and } \\
A_{j} \text { for one } j \neq i}} \varrho(\mathcal{B})+\sum_{\substack{\mathcal{B} \text { splits } 3 \text { parts of } \\
\mathcal{A}, \text { including } A_{i}}} \varrho(\mathcal{B})+\ldots\right) \\
& =\sum_{n \geqslant 1} n \sum_{\substack{\mathcal{B} \text { splits } \\
\text { parts of } \mathcal{A}}} \varrho(\mathcal{B})=\sum_{\mathcal{B} \in \mathbb{V}} \#(\mathcal{A}, \mathcal{B}) \varrho(\mathcal{B}),
\end{aligned}
$$

where the second to last step follows from a simple counting argument and $\#(\mathcal{A}, \mathcal{B})$ is the number of parts of $\mathcal{A}$ that are split by $\mathcal{B}$. If we now compare the two calculations and recall that $\varrho \geqslant 0$, the following result is immediate.

Lemma 1. Within a given lattice $\mathbb{V}$, the decay rates $\psi$ and $\chi$ satisfy the relation

$$
\psi(\mathcal{A})=\chi(\mathcal{A})+\sum_{n \geqslant 2}(n-1) \sum_{\substack{\mathcal{B} \text { splits } n \\ \text { parts of } \mathcal{A}}} \varrho(\mathcal{B}) .
$$

In particular, one has $\psi(\mathcal{A}) \geqslant \chi(\mathcal{A})$ for all $\mathcal{A} \in \mathbb{V}$.

Since $\psi(\underline{1})=\chi(\underline{1})$ whenever $\underline{1} \in \mathbb{V}$, Lemma 1 together with Fact 1 provides an alternative way to express the difference $\psi(\underline{1})-\psi(\mathcal{A})$ in this case as

$$
\psi(\underline{1})-\psi(\mathcal{A})=\sum_{\mathcal{A} \preccurlyeq \mathcal{B} \prec \underline{1}} \varrho(\mathcal{B})-\sum_{n \geqslant 2}(n-1) \sum_{\substack{\mathcal{B} \text { splits } n \\ \text { parts of } \mathcal{A}}} \varrho(\mathcal{B}) .
$$

This formula clearly shows that, in general, $\psi(\underline{1})-\psi(\mathcal{A})$ for $\mathcal{A} \neq \underline{1}$ can have either sign.

Let us now look more closely into the lattice $\mathbb{V}=\mathbb{I}(S)$, which is simpler than $\mathbb{P}(S)$, but still displays most of the relevant general phenomena.

\section{INTERVAL PARTITIONS}

This section deals with the case that $\mathbb{V}=\mathbb{I}(S)$, where $S$ is a fixed finite set, for instance $S=\{1,2, \ldots, n\}$.

Lemma 2. Let $S$ be as above, and assume that $\varrho^{S(\mathcal{C})}=0$ for all $\mathcal{C} \in \mathbb{I}(S)$ with $|\mathcal{C}|>2$. Then, for any $\varnothing \neq U \subseteq S$, one also has $\varrho^{U}(\mathcal{B})=0$ for all $\mathcal{B} \in \mathbb{I}(U)$ with $|\mathcal{B}|>2$. Moreover, $\psi^{U}(\mathcal{A})=\chi^{U}(\mathcal{A})$ holds for all $\varnothing \neq U \subseteq S$ and all $\mathcal{A} \in \mathbb{I}(U)$.

Proof. Since the induced (or marginalised) rates for $\mathcal{B} \in \mathbb{I}(U)$ are given by

$$
\varrho^{U}(\mathcal{B})=\sum_{\substack{\left.\mathcal{A} \in \mathbb{I}(S) \\ \mathcal{A}\right|_{U}=\mathcal{B}}} \varrho^{S}(\mathcal{A})
$$


the first claim is a simple consequence of the fact that, if $\mathcal{B} \in \mathbb{I}(U)$ with $|\mathcal{B}|>2$, any $\mathcal{A} \in \mathbb{I}(S)$ with $\left.\mathcal{A}\right|_{U}=\mathcal{B}$ must have at least three parts, too, due to the structure of the interval partitions.

When one considers the result of Lemma 1 for the lattice $\mathbb{V}=\mathbb{I}(U)$, one finds that each term in the difference $\psi^{U}(\mathcal{A})-\chi^{U}(\mathcal{A})$ is of the form $\varrho^{U}(\mathcal{B})$ for some $\mathcal{B} \in \mathbb{I}(U)$ with $|\mathcal{B}|>2$, hence vanishes by the first part of our proof.

For the generic choice of the recombination rates on $\mathbb{I}(S)$, our general solution has the form

$$
a_{t}^{S}(\mathcal{A})=\sum_{\mathcal{B} \succcurlyeq \mathcal{A}} \theta^{S}(\mathcal{A}, \mathcal{B}) \mathrm{e}^{-\psi^{S}(\mathcal{B}) t},
$$

with $\theta^{S}$ determined recursively as explained above. Let $\mu^{S}$ denote the Möbius function of the lattice $\mathbb{I}(S)$, which is given by $\mu^{S}(\mathcal{A}, \mathcal{B})=(-1)^{|\mathcal{A}|-|\mathcal{B}|}$ for $\mathcal{A} \preccurlyeq \mathcal{B}$ and $\mu^{S}(\mathcal{A}, \mathcal{B})=0$ otherwise. The definition of $\mu^{U}$ for $\mathbb{I}(U)$ with $\varnothing \neq U \subset S$ is analogous. These functions satisfy the following product identity.

Fact 2. Let $\mathcal{A}, \mathcal{B}, \mathcal{C} \in \mathbb{I}(S)$ with $\mathcal{A} \preccurlyeq \mathcal{B} \preccurlyeq \mathcal{C}$. Then, one has

$$
\mu^{S}(\mathcal{A}, \mathcal{B})=\prod_{i=1}^{r} \mu^{C_{i}}\left(\left.\mathcal{A}\right|_{C_{i}},\left.\mathcal{B}\right|_{C_{i}}\right)
$$

where $r=|\mathcal{C}|$, so $\mathcal{C}=\left\{C_{1}, C_{2}, \ldots, C_{r}\right\}$.

Proof. Since $\mathcal{A} \preccurlyeq \mathcal{C}$, we certainly have $|\mathcal{A}|=\sum_{i=1}^{r}|\mathcal{A}|_{C_{i}} \mid$, and analogously for $\mathcal{B}$. Now, one simply calculates

$$
\begin{aligned}
\prod_{i=1}^{r} \mu^{C_{i}}\left(\left.\mathcal{A}\right|_{C_{i}},\left.\mathcal{B}\right|_{C_{i}}\right) & =\prod_{i=1}^{r}(-1)^{\left.|\mathcal{A}|_{C_{i}}|-| \mathcal{B}\right|_{C_{i}} \mid}=(-1)^{\left.\sum_{i=1}^{r}|\mathcal{A}|_{C_{i}}|-| \mathcal{B}\right|_{C_{i}} \mid} \\
& =(-1)^{|\mathcal{A}|-|\mathcal{B}|}=\mu^{S}(\mathcal{A}, \mathcal{B}),
\end{aligned}
$$

which establishes the product formula.

With this preparation, one finds the following simplification of Eq. (12).

Proposition 1. Under the assumptions of Lemma 2, where $\varrho(\mathcal{C})=0$ for all $\mathcal{C} \in \mathbb{I}(S)$ with $|\mathcal{C}|>2$, the solution formula (12) simplifies to

$$
a_{t}^{S}(\mathcal{A})=\sum_{\mathcal{B} \succcurlyeq \mathcal{A}} \mu^{S}(\mathcal{A}, \mathcal{B}) \mathrm{e}^{-\chi^{S}(\mathcal{B}) t}
$$

with $\chi^{S}$ as above in Eq. (10). Moreover, this formula for $a_{t}^{S}$ holds for all remaining choices of the recombination rates.

Proof. In view of Lemma 2, it remains to prove that $\theta^{S}=\mu^{S}$. This will be done inductively again, so we will show that $\theta^{U}=\mu^{U}$ holds for all non-empty $U \subseteq S$.

When $|U|=1$, one has $\mathbb{I}(U)=\{\underline{1}\}$ and $\theta^{U}(\underline{1}, \underline{1})=1=\mu^{U}(\underline{1}, \underline{1})$. Likewise, when $|U|=2$, the corresponding lattice is $\mathbb{I}(U)=\{\underline{0}, \underline{1}\}$. It is clear that $\theta^{U}(\underline{0}, \underline{0})=\theta^{U}(\underline{1}, \underline{1})=1$, and one easily checks that $\theta^{U}(\underline{0}, \underline{1})=-1$ together with $\theta^{U}(\underline{1}, \underline{0})=0$, so $\theta^{U}=\mu^{U}$ in this case, too. 
Now, let us assume our claim to be true for all finite sets $U$ with cardinality $|U| \leqslant n$, and consider a set $S$ with $|S|=n+1$. We know that $\theta^{S}(\mathcal{A}, \mathcal{B})=0$ whenever $\mathcal{A} \npreceq \mathcal{B}$, so let $\mathcal{A}, \mathcal{B} \in \mathbb{I}(S)$ with $\mathcal{A} \preccurlyeq \mathcal{B}$ and assume first that $\mathcal{B} \prec \underline{1}$ (we will deal with the remaining case later). Now, our recursion together with the induction assumption and Lemma 2 gives us

$$
\begin{aligned}
\theta^{S}(\mathcal{A}, \mathcal{B}) & =\sum_{\mathcal{B} \preccurlyeq \mathcal{C} \prec \underline{1}} \frac{\varrho^{S}(\mathcal{C})}{\psi^{S}(\underline{1})-\psi^{S}(\mathcal{B})} \prod_{i=1}^{|\mathcal{C}|} \theta^{C_{i}}\left(\left.\mathcal{A}\right|_{C_{i}},\left.\mathcal{B}\right|_{C_{i}}\right) \\
& =\sum_{\mathcal{B} \preccurlyeq \mathcal{C} \prec \underline{1}} \frac{\varrho^{S}(\mathcal{C})}{\chi^{S}(\underline{1})-\chi^{S}(\mathcal{B})} \prod_{i=1}^{|\mathcal{C}|} \mu^{C_{i}}\left(\left.\mathcal{A}\right|_{C_{i}},\left.\mathcal{B}\right|_{C_{i}}\right) \\
& =\sum_{\mathcal{B} \preccurlyeq \mathcal{C} \prec \underline{1}} \frac{\varrho^{S}(\mathcal{C})}{\chi^{S}(\underline{1})-\chi^{S}(\mathcal{B})} \mu^{S}(\mathcal{A}, \mathcal{B})=\frac{\mu^{S}(\mathcal{A}, \mathcal{B})}{\chi^{S}(\underline{1})-\chi^{S}(\mathcal{B})} \sum_{\mathcal{B} \preccurlyeq \mathcal{C} \prec \underline{1}} \varrho^{S}(\mathcal{C}),
\end{aligned}
$$

where the second line follows because $\mathcal{C} \prec \underline{1}$ implies that all parts $C_{i}$ satisfy $\left|C_{i}\right| \leqslant n$, while the first step in the last line is a consequence of Fact 2 ,

Now, using the assumption and Lemma 2, the last sum evaluates as

$$
\sum_{\mathcal{B} \preccurlyeq \mathcal{C} \prec \underline{1}} \varrho^{S}(\mathcal{C})=\sum_{\mathcal{C} \neq \underline{1}} \varrho^{S}(\mathcal{C})-\sum_{\mathcal{D} \notin[\mathcal{B}, \underline{1}]} \varrho^{S}(\mathcal{D})=\chi^{S}(\underline{1})-\chi^{S}(\mathcal{B})
$$

wherefore we get $\theta^{S}(\mathcal{A}, \mathcal{B})=\mu^{S}(\mathcal{A}, \mathcal{B})$ for all $\mathcal{A} \preccurlyeq \mathcal{B} \prec \underline{1}$. Finally, since $\theta^{S}(\underline{1}, \underline{1})=1$ is automatic, we consider an arbitrary $\mathcal{A} \prec \underline{1}$ and find

$$
\theta^{S}(\mathcal{A}, \underline{1})=-\sum_{\mathcal{A} \preccurlyeq \mathcal{C} \prec \underline{1}} \theta^{S}(\mathcal{A}, \mathcal{C})=-\sum_{\mathcal{A} \preccurlyeq \mathcal{C} \prec \underline{1}} \mu^{S}(\mathcal{A}, \mathcal{C})
$$

from our previous argument. This finally also gives $\theta^{S}(\mathcal{A}, \underline{1})=\mu^{S}(\mathcal{A}, \underline{1})$ from the Möbius inversion formula.

The final claim is clear, because the solution now extends to the non-generic cases as well.

This result explicitly shows the compatibility of the general solution with the special case from [7, 6], which was also mentioned in [5]. What is more, it also shows that the linearity situation is slightly more general in the sense that one can still allow some recombination rates beyond the two parent case to be positive. Let us look into this point in more detail.

\section{Systems With up to FOUR SITES}

When we deal with $\mathbb{I}(S)$ for any non-empty finite set $S$ with up to 3 elements, we know that

$$
\psi^{S}=\chi^{S} \text { together with } \theta^{S}=\mu^{S} \text { and } \eta^{S}=\zeta^{S} .
$$

This means that the general solution is given by

$$
a_{t}^{S}(\mathcal{A})=\sum_{\mathcal{B} \succcurlyeq \mathcal{A}} \mu^{S}(\mathcal{A}, \mathcal{B}) \mathrm{e}^{-\chi^{S}(\mathcal{B}) t}
$$


for the initial condition $a_{0}^{S}(\mathcal{A})=\delta(\mathcal{A}, \underline{1})$, irrespective of the values of the rates $\varrho$. For $|S|=1$, this gives $a_{t} \equiv 1$ (we drop the upper index here), while $|S|=2$, meaning $\mathbb{I}(S)=\{\underline{1}, \underline{0}\}$, results in $\chi(\underline{1})=\varrho(\underline{0})$ and thus in the coefficient functions

$$
a_{t}(\underline{1})=\mathrm{e}^{-\varrho(\underline{0}) t} \quad \text { and } \quad a_{t}(\underline{0})=1-\mathrm{e}^{-\varrho(\underline{0}) t} .
$$

For $S=\{1,2,3\}$, one has $\underline{1}=(123), \underline{0}=(1|2| 3)$ and decay rates

\begin{tabular}{c|c}
$\mathcal{A}$ & $\chi$ \\
\hline$(123)$ & $\varrho(1 \mid 23)+\varrho(12 \mid 3)+\varrho(1|2| 3)$ \\
$(1 \mid 23)$ & $\varrho(12 \mid 3)+\varrho(1|2| 3)$ \\
$(12 \mid 3)$ & $\varrho(1 \mid 23)+\varrho(1|2| 3)$ \\
$(1|2| 3)$ & 0
\end{tabular}

The coefficient functions now read

$$
\begin{aligned}
a_{t}(\underline{1}) & =\mathrm{e}^{-\chi(\underline{1}) t}, \\
a_{t}(1 \mid 23) & =\mathrm{e}^{-\chi(1 \mid 23) t}-\mathrm{e}^{-\chi(\underline{1}) t}, \\
a_{t}(12 \mid 3) & =\mathrm{e}^{-\chi(12 \mid 3) t}-\mathrm{e}^{-\chi(\underline{1}) t}, \\
a_{t}(\underline{0}) & =1-\mathrm{e}^{-\chi(1 \mid 23) t}-\mathrm{e}^{-\chi(12 \mid 3) t}+\mathrm{e}^{-\chi(1) t} .
\end{aligned}
$$

Here, one can easily check that they indeed form a probability vector. Note that formula (13) also holds for $\mathbb{P}(S)$, then with the Möbius function $\mu$ and decay rate $\chi$ of $\mathbb{P}(S)$; see 5] for more. Note that the lattice $\mathbb{P}(S)$ contains one additional partition, namely $(13 \mid 2)$, in comparison to $\mathbb{I}(S)$. Up to this point, it is obvious that the probability vector $a_{t}$ also satisfies a linear ODE system - a point of view we will look at more closely in Section 7 below.

For $S=\{1,2,3,4\}$, things start to get more complicated. The lattice $\mathbb{I}(S)$, in obvious notation, is given by

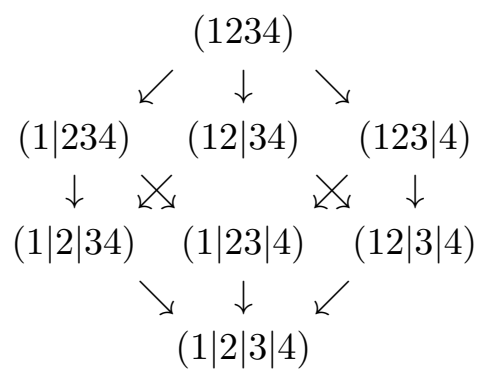

where an arrow points towards the next refined partition. An application of Lemma 1 to $\mathbb{V}=\mathbb{I}(S)$ gives the rate function as

$$
\psi(\mathcal{A})=\chi(\mathcal{A})+(\varrho(1|23| 4)+\varrho(\underline{0})) \delta(\mathcal{A},(12 \mid 34))
$$


with $\chi$ as in Eq. (10). An application of the recursion formula now results in the following expression for $\theta$, which we show in (upper triangular) matrix form for convenience,

\begin{tabular}{|c|c|c|c|c|c|c|c|c|}
\hline & 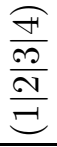 & 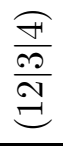 & $\begin{array}{l}\frac{\overparen{F}}{\mathscr{P}} \\
\stackrel{\stackrel{N}{\rightleftharpoons}}{\rightleftharpoons}\end{array}$ & $\frac{\overparen{F}}{\frac{\overparen{m}}{D}}$ & $\begin{array}{l}\stackrel{\overparen{F}}{\mathscr{T}} \\
\stackrel{\stackrel{Ð}{ت}}{\rightleftharpoons}\end{array}$ & 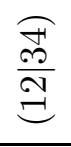 & 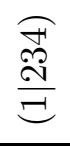 & 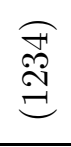 \\
\hline$(1|2| 3 \mid 4)$ & 1 & -1 & -1 & -1 & 1 & $x$ & 1 & $-x$ \\
\hline$(12|3| 4)$ & 0 & 1 & 0 & 0 & -1 & $-x$ & 0 & $x$ \\
\hline$(1|23| 4)$ & 0 & 0 & 1 & 0 & -1 & 0 & -1 & 1 \\
\hline$(1|2| 34)$ & 0 & 0 & 0 & 1 & 0 & $-x$ & -1 & $x$ \\
\hline$(123 \mid 4)$ & 0 & 0 & 0 & 0 & 1 & 0 & 0 & -1 \\
\hline$(12 \mid 34)$ & 0 & 0 & 0 & 0 & 0 & $x$ & 0 & $-x$ \\
\hline$(1 \mid 234)$ & 0 & 0 & 0 & 0 & 0 & 0 & 1 & -1 \\
\hline (1234) & 0 & 0 & 0 & 0 & 0 & 0 & 0 & 1 \\
\hline
\end{tabular}

Here, one has

$$
x=\frac{\varrho(12 \mid 34)}{\psi(\underline{1})-\psi(12 \mid 34)} \quad \text { with } \quad \psi(\underline{1})-\psi(12 \mid 34)=\varrho(12 \mid 34)-(\varrho(1|23| 4)+\varrho(1|2| 3 \mid 4)) .
$$

This clearly shows that $x$ can have either sign in general. When the denominator vanishes, we are outside the validity of the simple solution formula; see [5, Ch. 9] for details. We shall come back to this case below.

Before we expand on the solution, let us stick to the case that $x \neq 0$, where $\theta$ is invertible. The inverse function $\eta$ is still surprisingly simple, and given by $\eta(\mathcal{A}, \mathcal{B})=\zeta(\mathcal{A}, \mathcal{B})$ unless $\mathcal{A}=\mathcal{B}=(12 \mid 34)$, in which case one has

$$
\eta((12 \mid 34),(12 \mid 34))=\frac{1}{x}=\frac{\psi(\underline{1})-\psi(12 \mid 34)}{\varrho(12 \mid 34)}=1-\frac{\varrho(\underline{0})+\varrho(1|23| 4)}{\varrho(12 \mid 34)} .
$$

So, one sees that $\theta=\mu$ (and thus $\eta=\zeta$ ) happens precisely when $\psi=\chi$, which is equivalent with the condition $\varrho(\underline{0})=\varrho(1|23| 4)=0$.

Corollary 2. In the case of the lattice $\mathbb{I}(\{1,2,3,4\})$, we get $\psi(\mathcal{A})=\chi(\mathcal{A})$ for all $\mathcal{A}$ precisely when $\varrho(\underline{0})=\varrho(1|23| 4)=0$. Then, one also has $\theta=\mu$ together with $\eta=\zeta$, and the linear solution formula applies to all choices of the remaining recombination rates.

Now, since 4 is the smallest system size where $\theta$ can become non-invertible (which happens when $x=0$ ), we can go one step back and write down the ODE system for the functions $a_{t}(\mathcal{A})$ on $\mathbb{I}(S)$ explicitly as

$$
\dot{a}_{t}(\mathcal{A})=-\psi(\underline{1}) a_{t}(\mathcal{A})+\sum_{\mathcal{A} \preccurlyeq \mathcal{B} \prec \underline{1}} \mu(\mathcal{A}, \mathcal{B}) \sum_{\mathcal{B} \preccurlyeq \mathcal{C} \prec \underline{1}} \varrho(\mathcal{C}) \mathrm{e}^{-\psi(\mathcal{B}) t}
$$

where we have again dropped the upper index. This ODE follows from [5, Prop. 7] by restriction to the lattice $\mathbb{I}(S)$ and the insertion of the explicit solution for the possible subsystems, 
which have at most 3 sites and are thus given as above. Alternatively, one can use Eq. (6) for a direct derivation. With [5, Lemma 8], see also Fact 3 below, the solution of Eq. (16) is

$$
a_{t}(\mathcal{A})=a_{0}(\mathcal{A}) \mathrm{e}^{-\psi(\underline{1}) t}+\sum_{\mathcal{A} \preccurlyeq \mathcal{B} \prec \underline{1}} \mu(\mathcal{A}, \mathcal{B}) \sum_{\mathcal{B} \preccurlyeq \mathcal{C} \prec \underline{1}} \varrho(\mathcal{C}) E_{0}(\psi(\underline{1}), \psi(\mathcal{B}) ; t)
$$

with the function

$$
E_{0}(\alpha, \beta ; t)= \begin{cases}t \mathrm{e}^{-\alpha t}, & \text { if } \alpha=\beta, \\ \frac{1}{\alpha-\beta}\left(\mathrm{e}^{-\beta t}-\mathrm{e}^{-\alpha t}\right), & \text { otherwise. }\end{cases}
$$

Note that $E_{0}$ is continuous in the parameters, and symmetric under the exchange $\alpha \leftrightarrow \beta$.

Now, observing that $\psi(\underline{1})-\psi(\mathcal{A})=\chi(\underline{1})-\chi(\mathcal{A})$ for all $\mathcal{A} \neq(12 \mid 34)$ by Eq. (14) and using the first identity of Fact 1 , the solution simplifies to

$$
\begin{aligned}
a_{t}(\mathcal{A})= & a_{0}(\mathcal{A}) \mathrm{e}^{-\psi(\underline{1}) t}+\sum_{\substack{\mathcal{A} \preccurlyeq \mathcal{B} \prec 1 \\
\mathcal{B} \neq(12 \mid 34)}} \mu(\mathcal{A}, \mathcal{B})\left(\mathrm{e}^{-\psi(\mathcal{B}) t}-\mathrm{e}^{-\psi(\underline{1}) t}\right) \\
& +\mu(\mathcal{A},(12 \mid 34)) \varrho(12 \mid 34) E_{0}(\psi(\underline{1}), \psi(12 \mid 34) ; t),
\end{aligned}
$$

with $\varrho(12 \mid 34)=(\psi(\underline{1})-\psi(12 \mid 34))+\varrho(1|23| 4)+\varrho(\underline{0})$. The initial condition relevant to us is $a_{0}(\mathcal{A})=\delta(\mathcal{A}, \underline{1})$. Together with the upper Möbius summation, $\sum_{\mathcal{A} \preccurlyeq \mathcal{B} \preccurlyeq \underline{1}} \mu(\mathcal{A}, \mathcal{B})=\delta(\mathcal{A}, \underline{1})$, the last equation then further simplifies to

$$
a_{t}(\mathcal{A})=\sum_{\mathcal{B} \succcurlyeq \mathcal{A}} \mu(\mathcal{A}, \mathcal{B}) \mathrm{e}^{-\psi(\mathcal{B}) t}+\mu(\mathcal{A},(12 \mid 34))(\varrho(\underline{0})+\varrho(1|23| 4)) E_{0}(\psi(\underline{1}), \psi(12 \mid 34) ; t) .
$$

Here, when $\varrho(1|23| 4)+\varrho(\underline{0}) \neq 0$, the last factor becomes $t \mathrm{e}^{-\psi(\underline{1}) t}$ (via l'Hospital's rule, in agreement with the definition of $\left.E_{0}\right)$ when $\varrho(12 \mid 34) \rightarrow \varrho(1|23| 4)+\varrho(\underline{0})$, which means $\psi(12 \mid 34) \rightarrow \psi(\underline{1})$. This shows the appearance of a new function in the expansion when we hit one of the essential singularities of the recursive solution described earlier. Note that the full solution can still be extracted from the $\theta$-matrix, if one starts with a generic case and then rewrites the contributions with a prefactor $\pm x$ in terms of the function $E_{0}$.

In general, when $\varrho(1|23| 4)=\varrho(\underline{0})=0$, we are back in the situation of Corollary 2 , and thus in the realm of the linear scheme. Note that no restrictions occur for the values of $\varrho$ on $(1|2| 34)$ or $(12|3| 4)$, so that the linear scheme actually slightly extends beyond $\mathbb{I}_{2}(S)$. In any case, one has $a_{t}(\mathcal{A}) \geqslant 0$ for all $\mathcal{A} \in \mathbb{I}(S)$ and all $t \geqslant 0$, due to the preservation of positivity under the forward flow, together with $\sum_{\mathcal{A} \in \mathbb{I}(S)} a_{t}(\mathcal{A})=1$ for all $t \geqslant 0$, as a result of $\sum_{\mathcal{A} \in \mathbb{I}(S)} \mu(\mathcal{A},(12 \mid 34))=0$ and $\sum_{\mathcal{A} \preccurlyeq \mathcal{B}} \mu(\mathcal{A}, \mathcal{B})=\delta(\underline{0}, \mathcal{B})$ together with $\psi(\underline{0})=0$, which is nothing but a direct verification of the norm preservation mentioned in the Introduction.

Corollary 3. The solution of the recombination equation (4) on $\mathcal{M}(X)$, for $S=\{1,2,3,4\}$ with the lattice $\mathbb{V}=\mathbb{I}(S)$ and with a probability measure $\omega_{0} \in \mathcal{P}(X)$ as initial condition, is given by

$$
\omega_{t}=\sum_{\mathcal{A} \in \mathbb{I}(S)} a_{t}(\mathcal{A}) R_{\mathcal{A}}\left(\omega_{0}\right)
$$


with the convex coefficients $a_{t}(\mathcal{A})$ of $E q .(18)$.

\section{Recombination With interval Partitions For Five Sites}

Let us turn to the case of five sites, $S=\{1,2,3,4,5\}$. Here, as a result of Lemma 1, the decay rates are related by

$$
\psi(\mathcal{A})=\chi(\mathcal{A})+\sum_{\substack{\mathcal{B} \text { splits two } \\ \text { parts of } \mathcal{A}}} \varrho(\mathcal{B}),
$$

as a partition $\mathcal{A} \in \mathbb{I}(S)$ can have at most two splittable parts. In particular, $\psi(\mathcal{A})=\chi(\mathcal{A})$ for any $\mathcal{A}$ with at most one non-singleton part. For the other partitions, we get

\begin{tabular}{c|c}
$\mathcal{A}$ & $\psi(\mathcal{A})-\chi(\mathcal{A})$ \\
\hline$(12 \mid 345)$ & $\varrho(1|23| 45)+\varrho(1|234| 5)+\varrho(1|2| 3 \mid 45)+\varrho(1|2| 34 \mid 5)+\varrho(1|23| 4 \mid 5)+\varrho(\underline{0})$ \\
$(123 \mid 45)$ & $\varrho(12|34| 5)+\varrho(1|234| 5)+\varrho(12|3| 4 \mid 5)+\varrho(1|23| 4 \mid 5)+\varrho(1|2| 34 \mid 5)+\varrho(\underline{0})$ \\
$(1|23| 45)$ & $\varrho(12|34| 5)+\varrho(1|2| 34 \mid 5)+\varrho(12|3| 4 \mid 5)+\varrho(\underline{0})$ \\
$(12|3| 45)$ & $\varrho(1|234| 5)+\varrho(1|2| 34 \mid 5)+\varrho(1|23| 4 \mid 5)+\varrho(\underline{0})$ \\
$(12|34| 5)$ & $\varrho(1|23| 45)+\varrho(1|23| 4 \mid 5)+\varrho(1|2| 3 \mid 45)+\varrho(\underline{0})$
\end{tabular}

In particular, we get $\psi=\chi$ once again for a few more cases than those covered in Lemma 2 , because no conditions emerge for the rates $\varrho(1|2| 345), \varrho(12|3| 45)$ and $\varrho(123|4| 5)$.

Corollary 4. In the case of the lattice $\mathbb{I}(\{1,2,3,4,5\})$, we get $\psi(\mathcal{A})=\chi(\mathcal{A})$ for all $\mathcal{A}$ precisely when the eight recombination rates vanish that appear in Eq. (19). Then, one also has $\theta=\mu$ together with $\eta=\zeta$, and the linear solution formula applies to all choices of the remaining recombination rates.

The recursive formula for $\theta$ leads to the matrix shown in Table 1. Here, we used the shorthands

$$
x_{1}=\xi(12|34| 5), x_{2}=\xi(12|3| 45), x_{3}=\xi(1|23| 45), x_{4}=\xi(123 \mid 45) \text { and } x_{5}=\xi(12 \mid 345),
$$

where $\xi(\mathcal{A}):=\varrho\left(\mathcal{A}^{\prime}\right) /\left(\psi\left(\left.\underline{1}\right|_{\operatorname{supp}\left(\mathcal{A}^{\prime}\right)}\right)-\psi\left(\mathcal{A}^{\prime}\right)\right)$ with $\mathcal{A}^{\prime}$ being obtained from $\mathcal{A}$ by removing all singleton parts, so $(12|34| 5)^{\prime}=(12 \mid 34)$ and so on. In these expressions, the rates on subsystems are the induced rates according to Eq. (1). It is a somewhat surprising feature that the final expressions take a relatively simple, systematic form only after exploiting the recursive structure in this way. 
THE RECOMBINATION EQUATION FOR INTERVAL PARTITIONS

\begin{tabular}{|c|c|c|c|c|c|c|c|c|c|c|c|c|c|c|c|c|}
\hline & 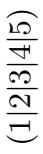 & $\frac{\frac{\partial D}{D}}{\frac{\frac{D}{D}}{\Xi}}$ & 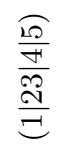 & $\frac{\frac{D}{D}}{\frac{m}{N}}$ & $\frac{\frac{\sqrt{\rho}}{9}}{\frac{\frac{9}{N}}{N}}$ & 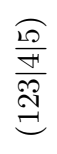 & 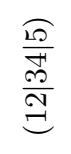 & $\frac{\widehat{\overparen{P}}}{\frac{\stackrel{\mathscr{P}}{\mathfrak{m}}}{\mathfrak{I}}}$ & 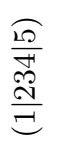 & 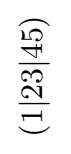 & 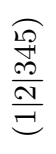 & $\begin{array}{l}\frac{\widehat{D D}}{\vec{N}} \\
\stackrel{\widetilde{N}}{=}\end{array}$ & 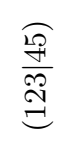 & 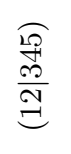 & 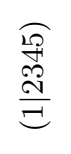 & 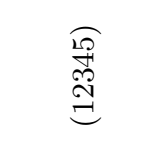 \\
\hline$(1|2| 3|4| 5)$ & 1 & -1 & -1 & -1 & -1 & 1 & $x_{1}$ & $x_{2}$ & 1 & $\overline{x_{3}}$ & 1 & $-x_{1}$ & $-x_{4}$ & $-x_{5}$ & $-x_{3}$ & $x_{5}+x_{4}-x_{2}$ \\
\hline$(12|3| 4 \mid 5)$ & 0 & 1 & 0 & 0 & 0 & -1 & $-x_{1}$ & $-x_{2}$ & 0 & 0 & 0 & $x_{1}$ & $x_{4}$ & $x_{5}$ & 0 & $x_{2}-x_{4}-x_{5}$ \\
\hline$(1|23| 4 \mid 5)$ & 0 & 0 & 1 & 0 & 0 & -1 & 0 & 0 & -1 & $-x_{3}$ & 0 & 1 & $x_{4}$ & 0 & $x_{3}$ & $-x_{4}$ \\
\hline$(1|2| 34 \mid 5)$ & 0 & 0 & 0 & 1 & 0 & 0 & $-x_{1}$ & 0 & -1 & 0 & -1 & $x_{1}$ & 0 & $x_{5}$ & 1 & $-x_{5}$ \\
\hline$(1|2| 3 \mid 45)$ & 0 & 0 & 0 & 0 & 1 & 0 & 0 & $-x_{2}$ & 0 & $-x_{3}$ & -1 & 0 & $x_{4}$ & $x_{5}$ & $x_{3}$ & $x_{2}-x_{4}-x_{5}$ \\
\hline$(123|4| 5)$ & 0 & 0 & 0 & 0 & 0 & 1 & 0 & 0 & 0 & 0 & 0 & -1 & $-x_{4}$ & 0 & 0 & $x_{4}$ \\
\hline$(12|34| 5)$ & 0 & 0 & 0 & 0 & 0 & 0 & $x_{1}$ & 0 & 0 & 0 & 0 & $-x_{1}$ & 0 & $-x_{5}$ & 0 & $x_{5}$ \\
\hline$(12|3| 45)$ & 0 & 0 & 0 & 0 & 0 & 0 & 0 & $x_{2}$ & 0 & 0 & 0 & 0 & $-x_{4}$ & $-x_{5}$ & 0 & $x_{5}+x_{4}-x_{2}$ \\
\hline$(1|234| 5)$ & 0 & 0 & 0 & 0 & 0 & 0 & 0 & 0 & 1 & 0 & 0 & -1 & 0 & 0 & -1 & 1 \\
\hline$(1|23| 45)$ & 0 & 0 & 0 & 0 & 0 & 0 & 0 & 0 & 0 & $x_{3}$ & 0 & 0 & $-x_{4}$ & 0 & $-x_{3}$ & $x_{4}$ \\
\hline$(1|2| 345)$ & 0 & 0 & 0 & 0 & 0 & 0 & 0 & 0 & 0 & 0 & 1 & 0 & 0 & $-x_{5}$ & -1 & $x_{5}$ \\
\hline$(1234 \mid 5)$ & 0 & 0 & 0 & 0 & 0 & 0 & 0 & 0 & 0 & 0 & 0 & 1 & 0 & 0 & 0 & -1 \\
\hline$(123 \mid 45)$ & 0 & 0 & 0 & 0 & 0 & 0 & 0 & 0 & 0 & 0 & 0 & 0 & $x_{4}$ & 0 & 0 & $-x_{4}$ \\
\hline$(12 \mid 345)$ & 0 & 0 & 0 & 0 & 0 & 0 & 0 & 0 & 0 & 0 & 0 & 0 & 0 & $x_{5}$ & 0 & $-x_{5}$ \\
\hline$(1 \mid 2345)$ & 0 & 0 & 0 & 0 & 0 & 0 & 0 & 0 & 0 & 0 & 0 & 0 & 0 & 0 & 1 & -1 \\
\hline (12345) & 0 & 0 & 0 & 0 & 0 & 0 & 0 & 0 & 0 & 0 & 0 & 0 & 0 & 0 & 0 & 1 \\
\hline
\end{tabular}

TABLE 1 . The $\theta$-matrix for the interval partitions of five sites.

If $x_{1} \cdot \ldots x_{5} \neq 0$, the function $\theta$ is invertible, and its inverse $\eta$ is given by

\begin{tabular}{|c|c|c|c|c|c|c|c|c|c|c|c|c|c|c|c|c|}
\hline & & & & & & & & & & & & & & & & \\
\hline$(1|2| 3|4| 5)$ & 1 & 1 & 1 & 1 & 1 & 1 & 1 & 1 & 1 & 1 & 1 & 1 & 1 & 1 & 1 & 1 \\
\hline$(12|3| 4 \mid 5)$ & 0 & 1 & 0 & 0 & 0 & 1 & 1 & 1 & 0 & 0 & 0 & 1 & 1 & 1 & 0 & \\
\hline$(1|23| 4 \mid 5)$ & 0 & 0 & 1 & 0 & 0 & 1 & 0 & 0 & 1 & 1 & 0 & 1 & 1 & 0 & 1 & \\
\hline$(1|2| 34 \mid 5)$ & 0 & 0 & 0 & 1 & 0 & 0 & 1 & 0 & 1 & 0 & 1 & 1 & 0 & 1 & 1 & 1 \\
\hline$(1|2| 3 \mid 45)$ & 0 & 0 & 0 & 0 & 1 & 0 & 0 & 1 & 0 & 1 & 1 & 0 & 1 & 1 & 1 & 1 \\
\hline$(123|4| 5)$ & 0 & 0 & 0 & 0 & 0 & 1 & 0 & 0 & 0 & 0 & 0 & 1 & 1 & 0 & 0 & 1 \\
\hline$(12|34| 5)$ & 0 & 0 & 0 & 0 & 0 & 0 & $\frac{1}{x_{1}}$ & 0 & 0 & 0 & 0 & 1 & 0 & $\frac{1}{x_{1}}$ & 0 & 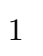 \\
\hline$(12|3| 45)$ & 0 & 0 & 0 & 0 & 0 & 0 & 0 & $\frac{1}{x_{2}}$ & 0 & 0 & 0 & 0 & $\frac{1}{x_{2}}$ & $\frac{1}{x_{2}}$ & 0 & 1 \\
\hline$(1|234| 5)$ & 0 & 0 & 0 & 0 & 0 & 0 & 0 & 0 & 1 & 0 & 0 & 1 & 0 & 0 & 1 & 1 \\
\hline$(1|23| 45)$ & 0 & 0 & 0 & 0 & 0 & 0 & 0 & 0 & 0 & $\frac{1}{x_{3}}$ & 0 & 0 & $\frac{1}{x_{3}}$ & 0 & 1 & 1 \\
\hline$(1|2| 345)$ & 0 & 0 & 0 & 0 & 0 & 0 & 0 & 0 & 0 & 0 & 1 & 0 & 0 & 1 & 1 & 1 \\
\hline$(1234 \mid 5)$ & 0 & 0 & 0 & 0 & 0 & 0 & 0 & 0 & 0 & 0 & 0 & 1 & 0 & 0 & 0 & 1 \\
\hline$(123 \mid 45)$ & 0 & 0 & 0 & 0 & 0 & 0 & 0 & 0 & 0 & 0 & 0 & 0 & $\frac{1}{x_{4}}$ & 0 & 0 & 1 \\
\hline$(12 \mid 345)$ & 0 & 0 & 0 & 0 & 0 & 0 & 0 & 0 & 0 & 0 & 0 & 0 & 0 & $\frac{1}{x_{5}}$ & 0 & 1 \\
\hline$(1 \mid 2345)$ & 0 & 0 & 0 & 0 & 0 & 0 & 0 & 0 & 0 & 0 & 0 & 0 & 0 & 0 & 1 & 1 \\
\hline (12345) & & 0 & 0 & 0 & 0 & 0 & 0 & 0 & 0 & 0 & 0 & 0 & 0 & 0 & 0 & 1 \\
\hline
\end{tabular}


While the $\theta$-coefficients suffice to write down the solution in the generic case, where we have $\psi^{U}(\underline{1}) \neq \psi^{U}(\mathcal{A})$ for all $\mathcal{A} \in \mathbb{I}(U)$ and all non-empty $U \subseteq S$, the degenerate cases require some care. First, for $\mathcal{A} \in\{\underline{1},(1 \mid 2345),(1234 \mid 5),(1|234| 5)\}$, the solution is always given by Eq. (13). These cases correspond to the four rows in the $\theta$-matrix of Table 1 with constant entries, which agree with the values of the Möbius function. For $\mathcal{A}=(12 \mid 345)$ and $\mathcal{A}=(123 \mid 45)$, the general solution reads

$$
a_{t}(\mathcal{A})=\varrho(\mathcal{A}) E_{0}(\psi(\underline{1}), \psi(\mathcal{A}) ; t)=\mathrm{e}^{-\psi(\underline{1}) t}-\mathrm{e}^{-\psi(\mathcal{A}) t}+(\psi(\mathcal{A})-\chi(\mathcal{A})) E_{0}(\psi(\underline{1}), \psi(\mathcal{A}) ; t)
$$

with the function $E_{0}$ from Eq. (17). Depending on the values of the recombination rates, one can thus pick up a term of the form $t \mathrm{e}^{-\psi(\mathcal{A}) t}$, as in the case of four sites. Next, one finds

$$
\begin{aligned}
& a_{t}(1|2| 345)=\mathrm{e}^{-\psi(1|2| 345) t}-\mathrm{e}^{-\psi(1 \mid 2345) t}-\varrho(12 \mid 345) E_{0}(\psi(\underline{1}), \psi(12 \mid 345) ; t), \\
& a_{t}(123|4| 5)=\mathrm{e}^{-\psi(123|4| 5) t}-\mathrm{e}^{-\psi(1234 \mid 5) t}-\varrho(123 \mid 45) E_{0}(\psi(\underline{1}), \psi(123 \mid 45) ; t),
\end{aligned}
$$

where the last term can be split as in the previous case. Similarly, one gets

$$
\begin{aligned}
a_{t}(12|3| 45)= & \varrho^{\prime}(12 \mid 45) E_{0}(\psi(\underline{1}), \psi(12|3| 45) ; t) \\
& -\varrho(123 \mid 45) E_{0}(\psi(\underline{1}), \psi(123 \mid 45) ; t)-\varrho(12 \mid 345) E_{0}(\psi(\underline{1}), \psi(12 \mid 345) ; t)
\end{aligned}
$$

with $\varrho^{\prime}(12 \mid 45)=\varrho(12|3| 45)+\varrho(123 \mid 45)+\varrho(12 \mid 345)$. Here and below, the ' indicates an induced quantity on a subsystem (here with four sites).

All remaining partitions are true refinements of (1|2345) or (1234|5), wherefore we may pick up a term of the form $t \mathrm{e}^{-\alpha t}$ already in the ODE, depending on the choice of parameters. To deal with this complication, let us first state a simple variant of the results in [5, Appendix].

Fact 3. Let $\rho$ as well as $\sigma_{1}, \ldots, \sigma_{m}$ and $\sigma_{1}^{\prime}, \ldots, \sigma_{n}^{\prime}$ be arbitrary non-negative numbers, and $\varphi$ a continuous function on $\mathbb{R}_{\geqslant 0}$ such that $\mathrm{e}^{\rho t} \varphi(t)$ is integrable. Then, the Cauchy problem defined by the $O D E$

$$
\dot{g}=-\rho g+\varphi(t)+\sum_{i=1}^{m} \varepsilon_{i}\left(\rho-\sigma_{i}\right) \mathrm{e}^{-\sigma_{i} t}+\sum_{j=1}^{n} \varepsilon_{j}^{\prime} \mathrm{e}^{-\sigma_{j}^{\prime} t}
$$

together with the initial condition $g(0)=g_{0}$ and $\varepsilon_{i}, \varepsilon_{j}^{\prime} \in \mathbb{R}$ has the unique solution

$$
g=g_{0} \mathrm{e}^{-\rho t}+\mathrm{e}^{-\rho t} \int_{0}^{t} \mathrm{e}^{\rho s} \varphi(s) \mathrm{d} s+\sum_{i=1}^{m} \varepsilon_{i}\left(\mathrm{e}^{-\sigma_{i} t}-\mathrm{e}^{-\rho t}\right)+\sum_{j=1}^{n} \varepsilon_{j}^{\prime} E_{0}\left(\rho, \sigma_{j}^{\prime} ; t\right),
$$

which holds for all $t \geqslant 0$.

Let us now consider the case $\mathcal{A}=(12|34| 5)$ in more detail. According to Eq. (6) , the ODE we have to solve here, after some calculations, reads

$$
\begin{aligned}
\dot{a}_{t}(\mathcal{A})= & -\psi(\underline{1}) a_{t}(\mathcal{A})+\sum_{\mathcal{A} \preccurlyeq \mathcal{B} \prec \underline{1}} \mu(\mathcal{A}, \mathcal{B})(\psi(\underline{1})-\psi(\mathcal{B})) \mathrm{e}^{-\psi(\mathcal{B}) t} \\
& +c E_{0}(\psi(1234 \mid 5), \psi(\mathcal{A}) ; t)+\sum_{\mathcal{A} \preccurlyeq \mathcal{B} \prec \underline{1}} \mu(\mathcal{A}, \mathcal{B})(\psi(\mathcal{B})-\chi(\mathcal{B})) \mathrm{e}^{-\psi(\mathcal{B}) t}
\end{aligned}
$$


where $c=\varrho(1234 \mid 5)\left(\varrho^{\prime}(1|2| 3 \mid 4)+\varrho^{\prime}(1|23| 4)\right)$. This is of the type covered by Fact 3, with $\varphi(t)=c E_{0}(\psi(1234 \mid 5), \psi(\mathcal{A}) ; t)$. Note that the two rates here generically differ from $\rho=\psi(\underline{1})$. Depending on the choice of the recombination rates, the solution can be a sum of exponentials (with coefficients given by $\theta$ ), which is the generic case. However, it can also contain a contribution of the form $t \mathrm{e}^{-\psi(\mathcal{B}) t}$ (when $\chi(\mathcal{B}) \neq \psi(\mathcal{B})=\psi(\underline{1})$ occurs) or even of the form $t^{2} \mathrm{e}^{-\psi(\mathcal{A}) t}$, which happens for the double degeneracy that $\psi(\mathcal{A})=\psi(1234 \mid 5)$ together with $\psi(\mathcal{A})=\psi(\underline{1})$, as a consequence of [5, Lemma 9]. We leave it to the reader to analyse the details of the various cases that are possible here. Clearly, the treatment of the partition $(1|23| 45)$ is completely analogous, so that we have covered all partitions with up to three parts.

The remaining five cases can be analysed in the same way. Doing so, one realises that the general form of the ODE for $a_{t}(\mathcal{A})$ with arbitrary $\mathcal{A} \in \mathbb{I}(S)$ reads

$$
\begin{aligned}
\dot{a}_{t}(\mathcal{A})= & -\psi(\underline{1}) a_{t}(\mathcal{A})+\sum_{\mathcal{A} \preccurlyeq \mathcal{B} \prec \underline{1}} \mu(\mathcal{A}, \mathcal{B})[(\psi(\underline{1})-\psi(\mathcal{B}))+(\psi(\mathcal{B})-\chi(\mathcal{B}))] \mathrm{e}^{-\psi(\mathcal{B}) t} \\
& +\mu(\mathcal{A},(12|34| 5)) c E_{0}(\psi(1234 \mid 5), \psi(12|34| 5) ; t) \\
& +\mu(\mathcal{A},(1|23| 45)) \tilde{c} E_{0}(\psi(1 \mid 2345), \psi(1|23| 45) ; t),
\end{aligned}
$$

with $c=\varrho(1234 \mid 5)\left(\varrho^{\prime}(1|2| 3 \mid 4)+\varrho^{\prime}(1|23| 4)\right)$ and $\tilde{c}=\varrho(1 \mid 2345)\left(\varrho^{\prime}(2|3| 4 \mid 5)+\varrho^{\prime}(2|34| 5)\right)$. The solution is fully covered by Fact [3, and the necessary case distinctions should be clear. In particular, the solutions will contain simple exponentials, contributions of type $E_{0}$ (whenever $\psi(\mathcal{B}) \neq \chi(\mathcal{B})$ in the sum, which leads to terms of the form $t \mathrm{e}^{-\alpha t}$ ), but possibly also integrals as in the previous two cases (with the same consequences, namely possible terms of the form $t^{2} \mathrm{e}^{-\alpha t}$ in the case of double degeneracies). Let us summarise this as follows.

Corollary 5. The solution of the general recombination equation (4) on $\mathcal{M}(X)$, for the set $S=\{1,2,3,4,5\}$ with the lattice $\mathbb{V}=\mathbb{I}(S)$ and with a probability measure $\omega_{0} \in \mathcal{P}(X)$ as initial condition, is given by

$$
\omega_{t}=\sum_{\mathcal{A} \in \mathbb{I}(S)} a_{t}(\mathcal{A}) R_{\mathcal{A}}\left(\omega_{0}\right)
$$

where the convex coefficients $a_{t}(\mathcal{A})$ are the unique solution of the Cauchy problem defined by Eq. (20) together with the initial condition $a_{0}(\mathcal{A})=\delta(\mathcal{A}, \underline{1})$, as given in Fact $\underline{3}$.

Beyond five sites, the complexity of the solution in the presence of degeneracies increases. In particular, for $n$ sites, one can in principle also obtain terms of the form $t^{m} \mathrm{e}^{-\alpha t}$ for any $0 \leqslant m \leqslant n-3$, though this requires an $m$-fold degeneracy in analogy to the appearance of double degeneracies in our above examples. Since the generic case without any of the 'bad' degeneracies most likely covers the practically relevant cases, we do not want to expand on this issue any further. We shall come back to it from a different point of view shortly. 


\section{FURTHER DIRECTIONS}

Let us return to the general case of $n$ sites, with an irreducible and indecomposable partition lattice $\mathbb{V}$. At this stage, we know that $\omega_{t}=\sum_{\mathcal{A} \in \mathbb{V}} a_{t}(\mathcal{A}) R_{\mathcal{A}}\left(\omega_{0}\right)$ solves the recombination ODE (4) when each $a_{t}(\mathcal{A})$ solves Eq. (6), both with the properly corresponding initial conditions. In the generic case with $a_{0}(\mathcal{A})=\delta(\mathcal{A}, \underline{1})$, this means $a_{t}(\mathcal{A})=\sum_{\mathcal{B} \succcurlyeq \mathcal{A}} \theta(\mathcal{A}, \mathcal{B}) \mathrm{e}^{-\psi(\mathcal{B}) t}$, where one also has $\mathrm{e}^{-\psi(\mathcal{B}) t}=\sum_{\mathcal{C} \succcurlyeq \mathcal{B}} \eta(\mathcal{B}, \mathcal{C}) a_{t}(\mathcal{C})$. This structure thus provides a mode decomposition (also known as decomposition into linkage disequilibria) $\omega_{t}=\sum_{\mathcal{A} \in \mathbb{V}} \nu_{t}(\mathcal{A})$ with

$$
\begin{aligned}
\nu_{t}(\mathcal{A}) & =\mathrm{e}^{-\psi(\mathcal{A}) t} \sum_{\mathcal{C} \preccurlyeq \mathcal{A}} \theta(\mathcal{C}, \mathcal{A}) R_{\mathcal{C}}\left(\omega_{0}\right) \\
& =\sum_{\mathcal{B} \succcurlyeq \mathcal{A}} \eta(\mathcal{A}, \mathcal{B}) a_{t}(\mathcal{B}) \sum_{\mathcal{C} \preccurlyeq \mathcal{A}} \theta(\mathcal{C}, \mathcal{A}) R_{\mathcal{C}}\left(\omega_{0}\right),
\end{aligned}
$$

where the (signed) measure $\nu_{t}(\mathcal{A})$ satisfies the linear ODE

$$
\dot{\nu}_{t}(\mathcal{A})=-\psi(\mathcal{A}) \nu_{t}(\mathcal{A})
$$

together with the initial condition $\nu_{0}(\mathcal{A})=\sum_{\mathcal{C} \preccurlyeq \mathcal{A}} \theta(\mathcal{C}, \mathcal{A}) R_{\mathcal{C}}\left(\omega_{0}\right)$.

Since our general assumptions on the recombination rates (for $\mathbb{V}$ being indecomposable and irreducible) imply that $\psi(\mathcal{A})>0$ for all $\mathcal{A} \neq \underline{0}$, we get the norm convergence

$$
\omega_{t} \stackrel{t \rightarrow \infty}{\longrightarrow} R_{\underline{0}}\left(\omega_{0}\right) \text {, }
$$

with exponential decay of all modes $\nu_{0}(\mathcal{A})$ for $\mathcal{A} \neq \underline{0}$. For any $\omega_{0} \in \mathcal{P}(X)$, there is thus the unique equilibrium, $R_{0}\left(\omega_{0}\right)$, and the convergence towards it is exponentially fast. In the degenerate cases, as explained above, this situation may have to be modified to include mode decay of the form $t^{m} \mathrm{e}^{-\alpha t}$ for some $m$, where $m \leqslant n-3$ with $n$ the number of sites, but this does not change the equilibrium. As the general structure should be clear, we leave further details to the reader.

Let us return to the generic case that $\psi^{U}(\underline{1}) \neq \psi^{U}(\mathcal{A})$ holds for all $\mathcal{A} \in \mathbb{V}(U)$ and all non-empty $U \subseteq S$. Here, we are in the regime of the recursive formula (9) for $\theta^{S}$. Though it seems difficult to write down a closed formula for $\theta^{S}$, we can say more on the diagonal elements. Define $\vartheta^{U}(\mathcal{A})=\theta^{U}(\mathcal{A}, \mathcal{A})$ and set $\vartheta^{\varnothing}(\varnothing)=1$. This way, $\vartheta$ is an element of the Möbius algebra, and satisfies the recursion

$$
\vartheta^{U}(\mathcal{A})=\sum_{\mathcal{A} \preccurlyeq \mathcal{B} \prec \underline{1}} \varrho^{U}(\mathcal{B}) \prod_{i=1}^{|\mathcal{B}|} \vartheta^{B_{i}}\left(\left.\mathcal{A}\right|_{B_{i}}\right),
$$

which is an immediate consequence of Eq. (9) and holds under the non-degeneracy conditions mentioned above. As introduced earlier, let $\mathcal{A}^{\prime}$ be the partition that emerges from $\mathcal{A}$ by removing all singleton parts, which includes the limiting case $\underline{0}^{\prime}=\varnothing$.

Proposition 2. Let $S$ be a finite set and $\mathbb{V}$ an indecomposable and irreducible lattice of partitions of $S$. For the generic choice of the recombination rates $\varrho^{S}$, the recursively defined 
functions $\vartheta^{U} \in \mathbb{M}_{\mathbb{R}}(\mathbb{V}(U))$ introduced above satisfy the relations

$$
\vartheta^{U}(\mathcal{A})=\vartheta^{\operatorname{supp}(\widetilde{\mathcal{A}})}(\widetilde{\mathcal{A}})
$$

for all $\mathcal{A} \in \mathbb{V}(U)$ and all non-empty $U \subseteq S$. Here, $\widetilde{A}$ is any partition that emerges from $\mathcal{A}$ by the removal of an arbitrary number of singletons, including the case $\widetilde{\mathcal{A}}=\mathcal{A}^{\prime}$.

Proof. The claim is true for all $\mathcal{A} \in \mathbb{P}(S)$ with any $S$ of cardinality $|S| \leqslant 3$ as a consequence of $\vartheta^{S}(\mathcal{A})=\mu^{S}(\mathcal{A}, \mathcal{A})=1$ for all $\mathcal{A} \in \mathbb{P}(S)$ in this case, hence also for the sublattice $\mathbb{V}$. The formula is also true for arbitrary $U \neq \varnothing$ when $\mathcal{A}$ contains no singletons or when $\widetilde{\mathcal{A}}=\mathcal{A}$ (both sides are equal) or when $\mathcal{A}=\underline{0}$ together with $\widetilde{\mathcal{A}}=\mathcal{A}^{\prime}=\varnothing$ (where both sides evaluate to 1 ).

To continue the proof by induction in the number of elements of $S$ respectively $U$, we may thus assume that $\mathcal{A}=\mathcal{D} \sqcup \mathcal{E}$ is the join of a partition $\mathcal{D}$, which may or may not contain one or more singletons, with another (non-empty) partition $\mathcal{E}$ that only contains singletons. With $D:=\operatorname{supp}(\mathcal{D}) \neq \varnothing$, we can then calculate

$$
\left(\psi^{S}(\underline{1})-\psi^{S}(\mathcal{A})\right) \vartheta^{S}(\mathcal{A})=\sum_{\mathcal{A} \preccurlyeq \mathcal{B} \prec \underline{1}} \varrho^{S}(\mathcal{B}) \prod_{i=1}^{|\mathcal{B}|} \vartheta^{B_{i}}\left(\left.\mathcal{A}\right|_{B_{i}}\right)=\sum_{\mathcal{F} \succcurlyeq \mathcal{D}} \sum_{\substack{\mathcal{C} \neq\left.\underline{1} \\ \mathcal{C}\right|_{D}=\mathcal{F}}} \varrho^{S}(\mathcal{C}) \prod_{i=1}^{|\mathcal{C}|} \vartheta^{C_{i}}\left(\left.\mathcal{A}\right|_{C_{i}}\right) .
$$

Using the induction hypothesis and the structure of $\mathcal{A}$, the last product can be simplified as

$$
\prod_{i=1}^{|\mathcal{C}|} \vartheta^{C_{i}}\left(\left.\mathcal{A}\right|_{C_{i}}\right)=\prod_{j=1}^{|\mathcal{F}|} \vartheta^{F_{j}}\left(\left.\mathcal{D}\right|_{F_{j}}\right)
$$

which allows to evaluate the inner sum over $\mathcal{C}$ as $\varrho^{D}(\mathcal{F})-\varrho^{S}(\underline{1}) \delta\left(\mathcal{F},\left.\underline{1}\right|_{D}\right)$. This gives

$$
\begin{aligned}
\left(\psi^{S}(\underline{1})-\psi^{S}(\mathcal{A})\right) \vartheta^{S}(\mathcal{A}) & =\left(\varrho^{D}\left(\left.\underline{1}\right|_{D}\right)-\varrho^{S}(\underline{1})\right) \vartheta^{D}(\mathcal{D})+\sum_{\mathcal{D} \preccurlyeq \mathcal{F} \prec \underline{1}} \varrho^{D}(\mathcal{F}) \prod_{j=1}^{|\mathcal{F}|} \vartheta^{F_{j}}\left(\left.\mathcal{D}\right|_{F_{j}}\right) \\
& =\left(\psi^{D}\left(\left.\underline{1}\right|_{D}\right)+\varrho^{D}\left(\left.\underline{1}\right|_{D}\right)-\varrho^{S}(\underline{1})-\psi^{D}(\mathcal{D})\right) \vartheta^{D}(\mathcal{D}) \\
& =\left(\psi^{S}(\underline{1})-\psi^{S}(\mathcal{A})\right) \vartheta^{S}(\mathcal{D}),
\end{aligned}
$$

where we used the recursion (21) as well as the identity $\psi^{D}(\mathcal{D})=\psi^{S}(\mathcal{A})$ and the relation between $\psi^{S}(\underline{1})$ and $\psi^{D}\left(\left.\underline{1}\right|_{D}\right)$, which both follow from the definition of the decay rates. Since $\psi^{S}(\underline{1}) \neq \psi^{S}(\mathcal{A})$ by assumption, our claim follows.

Note that $\vartheta$ coincides with the function $\xi$ used after Corollary 4 to calculate the recombination rate dependent terms in Table 1. Inspecting this table again together with the recursion for $\theta$ from Eq. (9), one sees that a similar reduction as for $\vartheta$ must be valid for $\theta$, too. Indeed, if $\mathcal{A} \preccurlyeq \mathcal{B}$, one can replace the pair $(\mathcal{A}, \mathcal{B})$ by a reduced one that emerges via the removal of all common singletons in $\mathcal{A}$ and $\mathcal{B}$. With $\theta^{\varnothing}(\varnothing, \varnothing):=1$, the reasoning of the proof of Proposition 2, applied to the recursion (9) for $\theta^{U}$, gives the following result. 
Theorem 3. Let $S$ and $\mathbb{V}$ be as in Proposition 2. For the generic choice of the recombination rates $\varrho^{S}$, the functions $\theta^{U} \in \mathbb{A}_{\mathbb{R}}(\mathbb{V}(U))$ satisfy the relations

$$
\theta^{U}(\mathcal{A}, \mathcal{B})=\theta^{\operatorname{supp}(\widetilde{\mathcal{B}})}\left(\left.\mathcal{A}\right|_{\operatorname{supp}(\widetilde{\mathcal{B}})}, \widetilde{\mathcal{B}}\right)
$$

for all $\mathcal{A}, \mathcal{B} \in \mathbb{V}(U)$ with $\mathcal{A} \preccurlyeq \mathcal{B}$ and all $\varnothing \neq U \subseteq S$. Here, $\widetilde{B}$ is any partition that emerges from $\mathcal{B}$ by the removal of an arbitrary number of singletons, including the case $\widetilde{\mathcal{B}}=\mathcal{B}^{\prime}$.

This reduction property helps to better understand the values of the $\theta$-coefficients and their recursive structure. Moreover, it also explains why and how the entries of the matrix in Eq. (15) reappear within Table 1 for five sites, once for partitions that comprise the singleton $\{5\}$ and once for those comprising $\{1\}$.

Invoking Lemma 3 from the Appendix and observing that, for $\mathcal{A} \preccurlyeq \mathcal{B}, \operatorname{chn}(\mathcal{A}, \mathcal{B})$ and $\operatorname{chn}\left(\left.\mathcal{A}\right|_{\operatorname{supp}\left(\mathcal{B}^{\prime}\right)}, \mathcal{B}^{\prime}\right)$ have the same cardinality, it is clear that the reduction property for $\theta$ extends to its inverse $\eta$ as follows.

Corollary 6. Let the assumption be as in Proposition 2 and Theorem 3, and assume that the recombination rates are chosen such that $\theta^{S} \in \mathbb{A}_{\mathbb{R}}(\mathbb{V})$ is well-defined and invertible, with inverse $\eta^{S}$. Then, one also has

$$
\eta^{S}(\mathcal{A}, \mathcal{B})=\eta^{\operatorname{supp}(\widetilde{\mathcal{B}})}\left(\left.\mathcal{A}\right|_{\operatorname{supp}(\widetilde{\mathcal{B}})}, \widetilde{\mathcal{B}}\right)
$$

for all $\mathcal{A}, \mathcal{B} \in \mathbb{V}$ with $\mathcal{A} \preccurlyeq \mathcal{B}$. As before, $\widetilde{B}$ is any partition that emerges from $\mathcal{B}$ by the removal of an arbitrary number of singletons, including the case $\widetilde{\mathcal{B}}=\mathcal{B}^{\prime}$.

Let us finally take a closer look at the structure of the probability vectors $a_{t}$ as a function of time, first in the simpler case that $\psi=\chi$, with $\varrho_{\text {tot }}:=\sum_{\mathcal{A} \in \mathbb{V}} \varrho(\mathcal{A})$.

Proposition 3. Let $S$ be a finite set and consider the lattice $\mathbb{I}(S)$. Moreover, assume that the recombination rates $\varrho(\mathcal{A})$ are chosen so that $\psi=\chi$ on $\mathbb{I}(S)$. Then, the solution $a_{t}$ of $E q$. (6) with initial condition $a_{0}(\mathcal{A})=\delta(\mathcal{A}, \underline{1})$ also solves the linear ODE system

$$
\begin{aligned}
\dot{a}_{t}(\mathcal{A}) & =-\sum_{\mathcal{B} \succcurlyeq \mathcal{A}} Q(\mathcal{A}, \mathcal{B}) a_{t}(\mathcal{B}) \quad \text { with } \\
Q(\mathcal{A}, \mathcal{B}) & =-\sum_{\mathcal{C} \in[\mathcal{A}, \mathcal{B}]} \mu(\mathcal{A}, \mathcal{C}) \chi(\mathcal{C})=-\varrho_{\text {tot }} \delta(\mathcal{A}, \mathcal{B})+\sum_{\mathcal{C} \succcurlyeq \mathcal{A}} \varrho(\mathcal{C}) \delta(\mathcal{A}, \mathcal{B} \wedge \mathcal{C}),
\end{aligned}
$$

where $Q$ is (the transpose of) a Markov generator, with the diagonal entries $Q(\mathcal{A}, \mathcal{A})=-\chi(\mathcal{A})$ being the eigenvalues of $Q$.

Proof. Under the assumptions, we know $a_{t}(\mathcal{A})=\sum_{\mathcal{C} \succcurlyeq \mathcal{A}} \mu(\mathcal{A}, \mathcal{C}) \mathrm{e}^{-\chi(\mathcal{C}) t}$ from Proposition 1 together with $\mathrm{e}^{-\chi(\mathcal{C}) t}=\sum_{\mathcal{B} \succcurlyeq \mathcal{C}} a_{t}(\mathcal{B})$ by Möbius inversion. Consequently, one has

$$
\begin{aligned}
\dot{a}_{t}(\mathcal{A}) & =-\sum_{\mathcal{C} \succcurlyeq \mathcal{A}} \mu(\mathcal{A}, \mathcal{C}) \chi(\mathcal{C}) \mathrm{e}^{-\chi(\mathcal{C}) t}=-\sum_{\mathcal{C} \succcurlyeq \mathcal{A}} \mu(\mathcal{A}, \mathcal{C}) \chi(\mathcal{C}) \sum_{\mathcal{B} \succcurlyeq \mathcal{C}} a_{t}(\mathcal{B}) \\
& =\sum_{\mathcal{B} \succcurlyeq \mathcal{A}}\left(-\sum_{\mathcal{C} \in[\mathcal{A}, \mathcal{B}]} \mu(\mathcal{A}, \mathcal{C}) \chi(\mathcal{C})\right) a_{t}(\mathcal{B})
\end{aligned}
$$


which proves the first claim and the first formula for $Q$.

Clearly, $Q(\mathcal{A}, \mathcal{B})=0$ whenever $\mathcal{A} \npreceq \mathcal{B}$. If $\mathcal{A} \preccurlyeq \mathcal{B}$, one uses Eq. (10) to get

$$
\begin{aligned}
Q(\mathcal{A}, \mathcal{B}) & =-\sum_{\mathcal{C} \in[\mathcal{A}, \mathcal{B}]} \mu(\mathcal{A}, \mathcal{C}) \chi(\mathcal{C})=-\sum_{\mathcal{C} \in[\mathcal{A}, \mathcal{B}]} \mu(\mathcal{A}, \mathcal{C})\left(\varrho_{\text {tot }}-\sum_{\mathcal{D} \in[\mathcal{C}, \underline{1}]} \varrho(\mathcal{D})\right) \\
& =-\varrho_{\text {tot }} \delta(\mathcal{A}, \mathcal{B})+\sum_{\mathcal{D} \succcurlyeq \mathcal{A}} \varrho(\mathcal{D}) \sum_{\mathcal{C} \in[\mathcal{A}, \mathcal{D}] \cap[\mathcal{A}, \mathcal{B}]} \mu(\mathcal{A}, \mathcal{C}) .
\end{aligned}
$$

Since $[\mathcal{A}, \mathcal{B}] \cap[\mathcal{A}, \mathcal{D}]=[\mathcal{A}, \mathcal{B} \wedge \mathcal{D}]$, the last sum is $\sum_{\mathcal{C} \in[\mathcal{A}, \mathcal{B} \wedge \mathcal{D}]} \mu(\mathcal{A}, \mathcal{C})=\delta(\mathcal{A}, \mathcal{B} \wedge \mathcal{D})$ and the second formula for $Q$ follows.

All off-diagonal terms of $Q$ are then clearly non-negative, while

$$
\sum_{\mathcal{A} \in \mathbb{I}(S)} Q(\mathcal{A}, \mathcal{B})=-\varrho_{\text {tot }}+\sum_{\mathcal{A} \preccurlyeq \mathcal{B}} \sum_{\mathcal{C} \succcurlyeq \mathcal{A}} \varrho(\mathcal{C}) \delta(\mathcal{A}, \mathcal{B} \wedge \mathcal{C})=-\varrho_{\text {tot }}+\sum_{\mathcal{C} \in \mathbb{I}(S)} \varrho(\mathcal{C}) \sum_{\mathcal{A} \preccurlyeq \mathcal{B} \wedge \mathcal{C}} \delta(\mathcal{A}, \mathcal{B} \wedge \mathcal{C})=0
$$

because $\mathcal{B} \wedge \mathcal{C}$ is a unique partition in $\mathbb{I}(S)$, so that the last sum is always 1 . This shows the claimed Markov generator property. The diagonal entries are clear from the first formula for $Q$. They are the eigenvalues because $Q$ is upper triangular.

Note that the second expression for $Q$ in Proposition 3 is slightly misleading in the sense that it suggests that all rates $\varrho(\mathcal{C})$ with $\mathcal{B} \wedge \mathcal{C}=\mathcal{A}$ contribute to $Q(\mathcal{A}, \mathcal{B})$. However, the condition $\psi=\chi$ forces $\varrho$ to vanish for many partitions as $|S|$ increases. In fact, as we shall see from Eq. (22) below, $Q(\mathcal{A}, \mathcal{B})>0$ is only possible if $\mathcal{A} \prec \mathcal{B}$ and if $\mathcal{A}$ refines precisely one part of $\mathcal{B}$. This is a somewhat surprising consequence of the underlying stochastic partitioning process discovered in [5, Sec. 6]. To explore this connection, one can now apply an analogous reasoning as in Proposition 3 to the general generic case, with the following result.

Theorem 4. Let $\mathbb{V}$ be an indecomposable and irreducible lattice of partitions of a finite set and assume that the recombination rates $\varrho(\mathcal{A})$ are such that $\theta \in \mathbb{A}_{\mathbb{R}}(\mathbb{V})$ is well-defined and invertible, with inverse $\eta$. Then, the solution $a_{t}$ of the ODE system (6) with initial condition $a_{0}(\mathcal{A})=\delta(\mathcal{A}, \underline{1})$ also solves the linear $O D E$ system

$$
\dot{a}_{t}(\mathcal{A})=-\sum_{\mathcal{B} \succcurlyeq \mathcal{A}} Q(\mathcal{A}, \mathcal{B}) a_{t}(\mathcal{B}) \quad \text { with } \quad Q(\mathcal{A}, \mathcal{B})=-\sum_{\mathcal{C} \in[\mathcal{A}, \mathcal{B}]} \theta(\mathcal{A}, \mathcal{C}) \psi(\mathcal{C}) \eta(\mathcal{C}, \mathcal{B}),
$$

where $Q$ is (the transpose of) a Markov generator. Moreover, $Q$ is upper triangular with diagonal entries $Q(\mathcal{A}, \mathcal{A})=-\psi(\mathcal{A})$, which are the eigenvalues of $Q$.

Proof. The first claim follows from Eq. (7) in conjunction with Theorem 2, The calculation runs as in the proof of Proposition 3, now with $\mathrm{e}^{-\psi(\mathcal{C}) t}=\sum_{\mathcal{B} \succcurlyeq \mathcal{C}} \eta(\mathcal{C}, \mathcal{B}) a_{t}(\mathcal{B})$ via the inversion formula for Eq. (17). Clearly, we have $Q(\mathcal{A}, \mathcal{B})=0$ whenever $\mathcal{A} \npreceq \mathcal{B}$, which implies that $Q$ is upper triangular. The diagonal entries are then the eigenvalues, and their values are a consequence of $\eta(\mathcal{A}, \mathcal{A})=1 / \theta(\mathcal{A}, \mathcal{A})$.

Next, for fixed $\mathcal{B} \in \mathbb{V}$, one finds the column sums

$$
\sum_{\mathcal{A} \in \mathbb{V}} Q(\mathcal{A}, \mathcal{B})=-\sum_{\mathcal{A} \preccurlyeq \mathcal{B}} \sum_{\mathcal{C} \in[\mathcal{A}, \mathcal{B}]} \theta(\mathcal{A}, \mathcal{C}) \psi(\mathcal{C}) \eta(\mathcal{C}, \mathcal{B})=\sum_{\mathcal{C} \preccurlyeq \mathcal{B}} \psi(\mathcal{C}) \eta(\mathcal{C}, \mathcal{B}) \sum_{\mathcal{A} \preccurlyeq \mathcal{C}} \theta(\mathcal{A}, \mathcal{C}) .
$$


Since the last sum is $\delta(\underline{0}, \mathcal{C})$ by [5, Prop. $8(2)$ ], which holds in this generality, one has

$$
\sum_{\mathcal{A} \in \mathbb{V}} Q(\mathcal{A}, \mathcal{B})=\psi(\underline{0}) \eta(\underline{0}, \mathcal{B})=0
$$

from $\psi(\underline{0})=0$, as is necessary for (the transpose of) a Markov generator. Since $Q$ is upper triangular, it remains to show that $Q(\mathcal{A}, \mathcal{B}) \geqslant 0$ whenever $\mathcal{A} \prec \mathcal{B}$.

To this end, we employ the underlying partitioning process from [5, Sec. 6]. Our coefficients $a_{t}(\mathcal{A})$ were identified as the first row of a Markov semigroup, namely as transition probabilities $\boldsymbol{P}_{t}(\underline{1} \rightarrow \mathcal{A})$. The corresponding Markov generator $\widetilde{Q}$ could be identified by also considering $\boldsymbol{P}_{t}(\mathcal{B} \rightarrow \mathcal{A})$ for an arbitrary $\mathcal{B} \in \mathbb{V}$, leading to the so-called Kolmogorov backward equation. By an application of [14, Thm. 2.1.1], we can switch to the corresponding forward equation with the same generator, thus giving a linear ODE system for the quantities $\boldsymbol{P}_{t}(\underline{\mathcal{A}} \rightarrow \mathcal{A})$ and hence $a_{t}(\mathcal{A})$ alone, which is nothing but the equation stated in the theorem, with $Q=\widetilde{Q}^{T}$. Consequently, all off-diagonal elements of $Q$ are non-negative.

Since it is difficult to calculate the off-diagonal elements of $Q$ from the formula in Theorem 4 , we employ [5, Thm. 3] to determine them. As mentioned earlier, $Q(\mathcal{A}, \mathcal{B})>0$ requires $\mathcal{A} \prec \mathcal{B}$ with $\mathcal{A}$ refining precisely one part of $\mathcal{B}$. So, we may assume this part to be $B_{1}$, hence $\mathcal{B}=\left\{B_{1}, B_{2}, \ldots, B_{r}\right\}$ with $r=|\mathcal{B}|$ and $\mathcal{A}=\left\{A_{1}, \ldots, A_{s}, B_{2}, \ldots, B_{r}\right\}$, with $s \geqslant 2$ and obvious meaning for $r=1$, where $|\mathcal{A}|=s+r-1$ and $\left.\mathcal{A}\right|_{B_{1}}=\left\{A_{1}, \ldots, A_{s}\right\}$. Then, we get

$$
Q(\mathcal{A}, \mathcal{B})=\varrho^{B_{1}}\left(\left.\mathcal{A}\right|_{B_{1}}\right)=\sum_{\substack{\left.\mathcal{C} \in \mathbb{V} \\ \mathcal{C}\right|_{B_{1}}=\left.\mathcal{A}\right|_{B_{1}}}} \varrho(\mathcal{C}),
$$

which clearly is always non-negative. One can now calculate that $\sum_{\mathcal{A} \prec \mathcal{B}} Q(\mathcal{A}, \mathcal{B})=\psi(\mathcal{B})$, in line with $Q$ having vanishing column sums. It is also instructive to calculate the matrix $Q$ for the lattice $\mathbb{V}=\mathbb{I}(\{1,2,3,4\})$ in both ways (via the formula from Theorem 4 and via Eq. (22)) to get an impression of the miraculous cancellations that happen along the way. The general verification of the equality of the two formulas is more difficult, and can partly be based on the reduction properties for $\theta$ and $\eta$, but also needs some lengthy calculations that we omit.

Now, the Markov generator $Q$ has a surprisingly simple form, and certainly no singularity of any kind. In fact, one can calculate it for $\mathbb{V}=\mathbb{P}(S)$, where it holds for all values of the recombination rates $\varrho$, and then restrict it to any sublattice, even to decomposable or reducible ones. The role of the somewhat tricky degeneracies appear in a new light then, too: Depending on the values of the parameters, the matrix $Q$ will or will not be diagonalisable. In the former case, we are in the regime where the recursive solution with the ansatz from Eq. (77) works. In the latter case, we have to employ the well-known theory of linear ODE systems for Jordan blocks, compare [1, Thm. 12.7] or [17, Ch. 17.VII], thus giving the appearance of terms of the form $t^{m} \mathrm{e}^{-\alpha t}$ another and more transparent meaning. Further ramifications of this connection will be explored in a separate publication [4]. 


\section{APPENDIX: AN INVERSION LEMMA FOR THE INCIDENCE ALGEBRA}

Let $L$ be a poset with ordering relation $\preccurlyeq$. We assume $L$ to be locally finite, which means that all intervals $[\mathcal{A}, \mathcal{B}]$ in $L$ are finite; compare [2, Chs. II and IV] for background. Now, let $\mathbb{A}_{K}(L)$ be the corresponding incidence algebra (over any field $K$ of characteristic 0 ). This is an associative $K$-algebra with the Kronecker function $\delta$ as its (two-sided) identity. Any element of the algebra, $\alpha$ say, satisfies $\alpha(\mathcal{A}, \mathcal{B})=0$ whenever $\mathcal{A}, \mathcal{B} \in L$ with $\mathcal{A} \npreceq \mathcal{B}$, and the multiplication of two elements is defined by

$$
(\alpha * \beta)(\mathcal{A}, \mathcal{B})=\sum_{\mathcal{C} \in[\mathcal{A}, \mathcal{B}]} \alpha(\mathcal{A}, \mathcal{C}) \beta(\mathcal{C}, \mathcal{B})
$$

An element $\alpha \in \mathbb{A}(L)$ is invertible if and only if $\alpha(\mathcal{A}, \mathcal{A}) \neq 0$ for all $\mathcal{A} \in L$. In this case, its unique inverse $\alpha^{-1}$ (which is both the left and the right inverse, so $\alpha^{-1} * \alpha=\alpha * \alpha^{-1}=\delta$ ) is recursively given by $\alpha^{-1}(\mathcal{A}, \mathcal{A})=1 / \alpha(\mathcal{A}, \mathcal{A})$ together with

$$
\alpha^{-1}(\mathcal{A}, \mathcal{B})=\frac{-1}{\alpha(\mathcal{A}, \mathcal{A})} \sum_{\mathcal{A} \prec \mathcal{D} \preccurlyeq \mathcal{B}} \alpha(\mathcal{A}, \mathcal{D}) \alpha^{-1}(\mathcal{D}, \mathcal{B})
$$

It is sometimes useful to have a closed expression for $\alpha^{-1}$ that is not recursive in nature. One classic identity, see [16, Thm. 3.3.22], uses a summation over chains. If $\mathcal{A} \preccurlyeq \mathcal{B}$, a chain from $\mathcal{A}$ to $\mathcal{B}$ is any increasing sequence $G \subseteq L$ of the form

$$
\mathcal{A}=\mathcal{G}_{1} \prec \mathcal{G}_{2} \prec \cdots \prec \mathcal{G}_{\ell}=\mathcal{B},
$$

where $|G|=\ell$ is called the length of $G$ (note that some authors use $|G|-1$ for the length). We use $\operatorname{chn}(\mathcal{A}, \mathcal{B})$ to denote the set of all chains from $\mathcal{A}$ to $\mathcal{B}$. When $\mathcal{A} \npreceq \mathcal{B}, \operatorname{chn}(\mathcal{A}, \mathcal{B})=\varnothing$ as usual.

Lemma 3. If $\alpha \in \mathbb{A}(L)$ is invertible, its inverse is given by

$$
\alpha^{-1}(\mathcal{A}, \mathcal{B})=\sum_{G \in \operatorname{chn}(\mathcal{A}, \mathcal{B})}(-1)^{|G|-1} \frac{\prod_{i=1}^{|G|-1} \alpha\left(\mathcal{G}_{i}, \mathcal{G}_{i+1}\right)}{\prod_{j=1}^{|G|} \alpha\left(\mathcal{G}_{j}, \mathcal{G}_{j}\right)}
$$

with the usual convention that an empty product is 1.

Proof. The claim can be established by induction on the maximal chain length in $\operatorname{chn}(\mathcal{A}, \mathcal{B})$. Clearly, the formula gives $\alpha^{-1}(\mathcal{A}, \mathcal{A})=1 / \alpha(\mathcal{A}, \mathcal{A})$, where $\operatorname{chn}(\mathcal{A}, \mathcal{A})$ consists of $G=\{\mathcal{A}\}$ only. If $\mathcal{A} \prec \mathcal{B}$ with the property that the maximal chain length between them is 2 , which means that $G=\{\mathcal{A}, \mathcal{B}\}$ is the only chain from $\mathcal{A}$ to $\mathcal{B}$, the formula gives

$$
\alpha^{-1}(\mathcal{A}, \mathcal{B})=-\frac{\alpha(\mathcal{A}, \mathcal{B})}{\alpha(\mathcal{A}, \mathcal{A}) \alpha(\mathcal{B}, \mathcal{B})}
$$

which clearly agrees with Eq. (23) for this case. 
Assume now that the formula holds for any pair with maximal chain length $k \leqslant n$, and consider $\mathcal{A} \prec \mathcal{B}$ with maximal chain length $n+1$. Then, Eq. (23) gives the relation

$$
\alpha^{-1}(\mathcal{A}, \mathcal{B})=-\sum_{\mathcal{A} \prec \mathcal{C} \preccurlyeq \mathcal{B}} \frac{\alpha(\mathcal{A}, \mathcal{C})}{\alpha(\mathcal{A}, \mathcal{A})} \alpha^{-1}(\mathcal{C}, \mathcal{B}) .
$$

Now, by construction, the maximal chain length between any $\mathcal{C}$ and $\mathcal{B}$ in the sum is bounded by $n$, so that we can use the induction hypothesis as follows,

$$
\begin{aligned}
\alpha^{-1}(\mathcal{A}, \mathcal{B}) & =-\sum_{\mathcal{A} \prec \mathcal{C} \preccurlyeq \mathcal{B}} \frac{\alpha(\mathcal{A}, \mathcal{C})}{\alpha(\mathcal{A}, \mathcal{A})} \sum_{G \in \operatorname{chn}(\mathcal{C}, \mathcal{B})}(-1)^{|G|-1} \frac{\prod_{i=1}^{|G|-1} \alpha\left(\mathcal{G}_{i}, \mathcal{G}_{i+1}\right)}{\prod_{j=1}^{|G|} \alpha\left(\mathcal{G}_{j}, \mathcal{G}_{j}\right)} \\
& =\sum_{\{\mathcal{A}\} \cup G \in \operatorname{Ghn}(\mathcal{A}, \mathcal{B})}(-1)^{|G|} \frac{\alpha\left(\mathcal{A}, \mathcal{G}_{1}\right)}{\alpha(\mathcal{A}, \mathcal{A})} \frac{\prod_{i=1}^{|G|-1} \alpha\left(\mathcal{G}_{i}, \mathcal{G}_{i+1}\right)}{\prod_{j=1}^{|G|} \alpha\left(\mathcal{G}_{j}, \mathcal{G}_{j}\right)} \\
& =\sum_{H \in \operatorname{chn}(\mathcal{A}, \mathcal{B})}(-1)^{|H|-1} \frac{\prod_{i=1}^{|H|-1} \alpha\left(\mathcal{H}_{i}, \mathcal{H}_{i+1}\right)}{\prod_{j=1}^{|H|} \alpha\left(\mathcal{H}_{j}, \mathcal{H}_{j}\right)}
\end{aligned}
$$

where the calculation really is a consequence of how each chain from $\mathcal{A}$ to $\mathcal{B}$ can uniquely be decomposed into one step from $\mathcal{A}$ to $\mathcal{C}$, with $\mathcal{A} \prec \mathcal{C} \preccurlyeq \mathcal{B}$, and a chain from $\mathcal{C}$ to $\mathcal{B}$.

\section{ACKNOWLEDGEMENTS}

It is our pleasure to thank E. Baake and M. Salamat for valuable discussions. ES is grateful to the FSPM ${ }^{2}$ of Bielefeld University for financial support during her stay in Bielefeld. This work was also supported by the German Research Foundation (DFG), within the SPP 1590.

\section{REFERENCES}

[1] H. Amann, Gewöhnliche Differentialgleichungen, 2nd ed., de Gryuter, Berlin (1995).

[2] M. Aigner, Combinatorial Theory, reprint, Springer, Berlin (1997).

[3] E. Baake, Deterministic and stochastic aspects of single-crossover recombination, in: Proceedings of the International Congress of Mathematicians, Hyderabad, India, 2010, Vol. VI, ed. J. Bhatia, Hindustan Book Agency, New Delhi (2010), pp. 3037-3053; arXiv:1101.2081.

[4] E. Baake and M. Baake, Haldane linearisation done right: Solving the nonlinear recombination equation the easy way, Discr. Cont. Dynam. Syst. A 36 (2016) 6645-6656; arXiv:1606.05175.

[5] E. Baake, M. Baake and M. Salamat, The general recombination equation in continuous time and its solution, Discr. Cont. Dynam. Syst. A 36 (2016) 63-95; arXiv:1409.1378.

[6] M. Baake, Recombination semigroups on measure spaces, Monatsh. Math. 146 (2005) 267-278 and 150 (2007) 83-84 (Addendum); arXiv:math.CA/0506099.

[7] M. Baake and E. Baake, An exactly solved model for mutation, recombination and selection, Can. J. Math. 55 (2003) 3-41 and 60 (2008) 264-265 (Erratum); arXiv:math.CA/0210422.

[8] J.H. Bennett, On the theory of random mating, Ann. Human Gen. 18 (1954) 311-317. 
[9] R. Bürger, The Mathematical Theory of Selection, Recombination and Mutation, Wiley, Chichester (2000).

[10] K.J. Dawson, The evolution of a population under recombination: How to linearise the dynamics, Lin. Alg. Appl. 348 (2002) 115-137.

[11] R. Durrett, Probability Models for DNA Sequence Evolution, 2nd ed., Springer, New York (2008).

[12] H. Geiringer, On the probability theory of linkage in Mendelian heredity, Ann. Math. Stat. 15 (1944) 25-57.

[13] Y.I. Lyubich, Mathematical Structures in Population Genetics, Springer, Berlin (1992).

[14] J.R. Norris, Markov Chains, Cambridge University Press, Cambridge (1997), reprint (2005).

[15] E. Popa, Some remarks on a nonlinear semigroup acting on positive measures, in: Applied Analysis and Differential Equations, O. Carja and I.I. Vrabie (eds.), World Scientific, Singapore (2007), pp. 308-319.

[16] E. Spiegel and C.J. O’Donnell, Incidence Algebras, Marcel Dekker, New York (1997).

[17] W. Walter, Ordinary Differential Equations, GTM 182, Springer, New York (1998).

Fakultät für Mathematik, Universität Bielefeld, Postfach 100131, 33501 Bielefeld, Germany

Department of Applied Mathematics, School of Mathematical Sciences,

Ferdowsi University of Mashhad (FUM), Mashhad, Iran 